\title{
Rechtsgeschichte
}

http://www.rg-rechtsgeschichte.de/rg9

$\operatorname{Rg} 2006 \quad 12-35$

Zitiervorschlag: Rechtsgeschichte Rg 9 (2006)

http://dx.doi.org/10.12946/rg09/012-035

\section{Ursula LehmkuhI}

\section{Ein Kristallpalast für New York}

Kulturtransfer und nationale Identitätskonstruktion in den USA vor dem Bürgerkrieg 


\section{Abstract}

On July 14, 1853 the second World Exhibition opened its gates in New York. Regarding architecture and themes a simple transfer of the London concept was intended. The shift from London to New York was based on a series of more or less successful translations and transfer-processes. The concept of World Exhibition had to be adapted according to organisation, administration, town planning and in general according to the social, economic and cultural settings of the young republic of the USA. The essay analyzes how processes of adaptation worked, which essential changes they produced and how successful they were in the end. Therefore it works out the specific example of a European-American transfer-process in the 19th century and intends to underline the inherent laws and specific paths of development of such processes. 


\section{Ein Kristallpalast für New York}

Kulturtransfer und nationale Identitätskonstruktion in den USA vor dem Bürgerkrieg

Am 26. Dezember I 85 I, zwei Monate nachdem der Kristallpalast in London, in dem die erste Weltausstellung stattfand, seine Tore schloss, fasste das Board of Alderman der City of New York den Beschluss, eine Industrial Exhibition of all Nations in New York durchzuführen. Zu diesem Zweck sollte am Madison Square ein Gebäude aus Stahl und Glas errichtet werden. Das Gebäude sollte "good, strong and handsome « sein und wie der Londoner Kristallpalast nach zwei Jahren wieder abgerissen werden. Die New Yorker Kristallpalastausstellung wurde am I4. Juli I853 vom amerikanischen Präsidenten Franklin Pierce mit großem Zeremoniell eröffnet und zählte rund 5 ooo Aussteller, von denen etwa die Hälfte aus den USA kamen. Jedoch blieben die Besucherzahlen ebenso hinter den Erwartungen zurück wie die Einnahmen. Der Veranstaltung gelang es nicht, eine ausreichende internationale Beteiligung zu mobilisieren. Als die Schau am I. November I 854 ihre Pforten schloss, hatte die von einem privaten Unternehmen organisierte und finanzierte Weltausstellung ein Defizit von 300000 Dollar erwirtschaftet. ${ }^{\mathbf{I}}$ Den New Yorker Glaspalast ereilte dasselbe Schicksal, wie es auch sein Londoner Vorbild ein dreiviertel Jahrhundert später ereilen sollte: Anfang Oktober I 858 zerstörte ein spektakuläres Feuer die Eisen-Glas-Konstruktion.

Bereits Ende August I85I, noch während der Londoner Weltausstellung, hatte Edward Riddle, Kaufmann aus Boston und Beauftragter für die amerikanische Ausstellung in London, beeindruckt von dem überwältigenden Erfolg der amerikanischen Ausstellungsgegenstände, angekündigt, dass er einen Kristallpalast in New York bauen wolle. ${ }^{2}$ Viele Ausstellungsgegenstände wurden deshalb erst gar nicht wieder in ihre Herkunftsländer zurückgeführt, sondern blieben gut verpackt in London und warteten dort auf den Weitertransport nach New York. Die geplante Weltausstellung in den USA intendierte keine inhaltliche Weiterentwicklung des Ausstellungskonzeptes. Dies war auch angesichts der zeitlichen Nähe zwischen der Londoner und der New Yorker Ausstellung kaum zu erwarten. ${ }^{3}$ Vielmehr sollten der amerikanischen Bevölkerung die Errungenschaften des technischen Fortschritts präsentiert werden. Trotz der für das Jahr I $85 \mathrm{I}$ beeindruckenden Besucherzahl von über sechs Millionen war die Londoner Ausstellung im Hinblick auf das Publikum doch letztlich eine europäische Veranstaltung geblieben. Reisen zwischen den Kontinenten waren um die Jahrhundertmitte noch so beschwerlich und kostspielig, dass sie prohibitiv auf die Teilnahme amerikanischer Besucher wirkten. ${ }^{4}$

Auch wenn im Hinblick auf Architektur und Inhalt eine schlichte Übertragung - man könnte fast von Verpflanzung sprechen - der Londoner Ausstellung in die Neue Welt intendiert war, ${ }^{5}$ basierte die Umsetzung des Unternehmens auf einer ganzen Reihe von mehr oder weniger geglückten kulturellen Übersetzungsund Transferprozessen. Das Konzept > Weltausstellung musste an die organisatorischen, verwaltungsstrukturellen, städteplanerischen und allgemein an die sozialen, wirtschaftlichen und
I 300000 Dollar im Jahr I 854 entsprechen ca. 7 Millionen Dollar heute.

2 Nicht nur in London, sondern auch in New York wurde überschwänglich von der »superiority « der jungen amerikanischen Nation berichtet. C. T. Rodgers, American Superiority at the World's Fair, Philadelphia I 852 ; URSULA LEHMKUHL, Una mietitrice come catalizzatore. La Great Exhibition

응 del I 85 I e la costruzione sociale della relazione speciale angloamericana (2004) I4I-64.

$3 \mathrm{Vgl}$. hierzu auch die kritischen Kommentare des Scientific American, der aufgrund der zeitlichen Nähe zur Londoner Ausstellung und weil aus diesem Grund keine spektakulären Neuerungen zu erwarten waren, die Organisation einer Weltausstellung in New York zunächst ablehnte. Der Scientific American war I 845 gegründet worden und stellte das erste amerikanische Wissenschaftsmagazin dar, das wöchentlich mit einem Umfang von acht vierspaltigen Seiten erschien.

4 Markus Günther, Auf dem Weg in die Neue Welt (2005).

5 Ein zeitgenössischer Beobachter kommentierte: "The exhibition does not ... propose to be a rival, but a continuation of the former, and the glory which attached to the one will be transferred to the other «, New York Daily Times, Jan. 3I, I852, 7 . 
kulturellen Rahmenbedingungen der jungen Republik angepasst werden. Wie diese Anpassungsprozesse abliefen, zu welchen inhaltlichen Veränderungen sie führten und wie erfolgreich sie letztlich waren, soll am Beispiel der New Yorker Kristallpalastausstellung von I 853 vorgestellt werden.

Die Analyse der Transferprozesse erfolgt entlang der drei zentralen Funktionen von Weltausstellungen im I9. Jahrhundert: Erstens und vor allem waren Weltausstellungen im Zeitalter des aufkommenden Nationalismus trotz bzw. parallel zum völkerverbindenden Anspruch des bürgerlichen Internationalismus ein Medium und Instrument staatlicher Prestigepolitik. In kaum einem anderen Medium wird die für das I9. Jahrhundert typische nationalistische Überformung des Universalismus deutlicher. ${ }^{6}$ Weltausstellungen sind letztlich »exercises in the imagery of nationalism «. ${ }^{7}$ Gleichzeitig sind Konzeption, Ziele und Format der Weltausstellungen Ausdruck des nationenübergreifenden bürgerlichen Zeitgeistes und der ihn prägenden philosophischen Denkschulen, die letztlich im Internationalismus des I9. Jahrhunderts konvergierten: Kosmopolitismus, Pazifismus, Liberalismus und Utilitarismus bildeten die ideellen Folien für die Weltausstellung. Der friedliche Wettbewerb der Nationen, der sich letztlich als Wettstreit der Kulturen darstellte, sollte zum Wohle der ganzen Menschheit den zivilisatorischen Fortschritt befördern. In kaum einem anderen zeitgenössischen Medium findet man eine derart prononcierte internationalistische Überhöhung nationalistischer Ziele und Interessen. Weltausstellungen lieferten als kommunikative Vermittlungsinstanzen einen Beitrag zur politischen Integration im jeweiligen Gastgeberland, aber auch zwischen den beteiligten Nationen. ${ }^{8}$ Sie trugen damit zur Strukturierung und Transformation weltgesellschaftlicher Beziehungen bei, und zwar gerade auch deshalb, weil sie als Medium nationaler Selbstdarstellung und zur Projektion der eigenen Macht und Größe nach außen dienten.

Weltausstellungen waren zweitens ein urbanes Phänomen, das eng an die Entwicklung moderner Großstädte gekoppelt war. Weltausstellungen schufen öffentliche Räume, die maßgeblichen Einfluss auf die sich im Verlaufe des I9. Jahrhunderts herausbildende kommunikative Interdependenz zwischen metropolitanem und globalem Raum nahmen. Weltausstellungen konstituierten damit ein Phänomen, das gewöhnlicherweise als Element des Globalisierungsprozesses des späten 20. Jahrhunderts herausgestellt wird: die »global city «. ${ }^{9}$ Im öffentlichen Raum der Weltausstellung kommunizierten Besucher, Berichterstatter, gesellschaftliche Gruppen und Staaten miteinander, verhandelten dabei gemeinsame Entwicklungsfragen der Menschheit und artikulierten Zukunftsdiskurse. Auf engstem Raum wurden Politik und Wirtschaft, Wissenschaft und Technik, Bildung, Kunst und Unterhaltung zu einem vielschichtigen Beziehungsgeflecht vereint. Der Fortschritt der Menschheit sollte auf systematische und vergleichende Weise präsentiert und ein möglichst umfassendes Bild der zivilisatorisch-kulturellen Entwicklung gezeichnet werden. ${ }^{\text {Io }}$

Drittens waren Weltausstellungen ein Instrument der Bildung und des Fortschritts und ein zentrales Symbol der Moderne, die sich in der Architektur u.a. in rationalen Konstruktionsprinzipien und serieller Produktion niederschlug. ${ }^{\text {II }}$ Das architektonische Konzept des Kristall- oder Industriepalastes, das bis zur Pariser Weltausstellung I 867 die Weltausstellungsarchitektur dominierte und erst danach vom Konzept des Ausstellungsparks abgelöst wurde, visualisierte dabei nicht nur Modernität und
6 Dieter Langewiesche, Nation, Nationalismus, Nationalstaat in Deutschland und Europa (2000) 35 .

7 Martin Wörner, Die Welt an einem Ort (2000) 4; vgl. auch UTZ Haltern, Die »Welt als Schaustellung «( I973a) I-40; BuRTON BENEDICT, International exhibitions and national identity (I99I) 5-9.
8 Wolfram Kaiser, Die Welt im Dorf (2000) 3-IO.

9 Saskia Sassen, Cities in a world economy (I994); DIES., The global city (200I); DIEs., Global networks, linked cities (2002).

io Winfried Kretschmer, Geschichte der Weltausstellungen (I999).

I I Roland Marchand, Corporate Imagery and Popular Education (I99I) I 8; ROBERT W. RYDELL,
All the world's a fair (I993); DERs., The Open (Laboratory) Door. Scientists and Mass Culture (i988) 6i; Selim H. Peabody, World's fairs (I902). 
Fortschritt, sondern zugleich die Aufhebung der Grenzen zwischen innen und außen, zwischen lokaler Präsentation und globaler Repräsentation. Glasarchitektur bedeutete Leichtigkeit, Durchsichtigkeit und gewagte Strukturen. Sie war die architektonische Übersetzung sozialer Utopien und rettete - jedenfalls in der europäischen Tradition des Kristallpalastes - den Geist der Romantik in die Moderne hinüber. Neben der Visualisierung und Inszenierung des Fortschrittsdiskurses lieferte die frühe Weltausstellungsarchitektur in der Form des Kristallpalastes die metaphorische Grundlage für die Kommunikation eines breiten Spektrums von Themen, die in der europäischen Tradition mit der kristallinen Form assoziiert bzw. in dieser erblickt wurden. ${ }^{\text {I2 }}$

Diese drei im europäischen Kontext gewachsenen Funktionen von Industrie- und Weltausstellungen sollen als zentrale Hintergrundbedingungen die Analyse der ersten amerikanischen Weltausstellung von I 853 leiten. Empirisch betritt die Untersuchung damit in mindestens zweierlei Hinsicht Neuland. ${ }^{13}$ Zum einen hat sich weder die amerikanische noch die europäische Forschung bislang intensiver mit der ersten Weltausstellung in den USA beschäftigt. Für Winfried Kretschmer etwa wird die Geschichte der Kristallpaläste mit den beiden Daten I 85 I: London und I 855 : Paris markiert. ${ }^{\mathrm{I} 4}$ Und auch für Ilja Mieck figuriert die Weltausstellung von I 855 als die "zweite Weltausstellung", obwohl die Entscheidung der Franzosen, eine Weltausstel- lung in Paris auszurichten und zu diesem Zweck einen Kristallpalast zu errichten, ein knappes dreiviertel Jahr später fiel als die der Amerikaner und auch die Ausstellung selbst erst zwei Jahre nach der Weltausstellung in New York stattfand. Aus der Feder amerikanischer Historiker liegen einige wenige Aufsätze ${ }^{15}$ und ein Eintrag im Historical Dictionary of World's Fairs and Expositions ${ }^{\mathrm{I} 6}$ vor, die allerdings, im Falle von Hirschfelds und Steens Beiträgen aus den Jahren I957 und I963, aus der teleologischen Perspektive des master narrative der I 95 oer Jahre - Aufstieg der USA zur Weltmacht - geschrieben sind, während Post vor allem die technikgeschichtlichen Aspekte abdeckt. Sowohl im Hinblick auf die vergleichend angelegte monographische Literatur als auch hinsichtlich transfergeschichtlicher Fragestellungen bleibt die Weltausstellung I 853 ein blinder Fleck. Für die Zeitgenossen war sie allerdings ein großes Ereignis. In zahlreichen Abhandlungen und Reminiszenzen wird über sie berichtet. ${ }^{17}$ Die Weltausstellung in New York galt als Auftakt zu einer »important era in the history of manufacturing and producing industry in the United States ${ }^{\text {I }}{ }^{\text {8 }}$ Der Impuls, der von der Ausstellung für den »mechanical and artistic genius of the country « ausging, sei allerdings - so die New York Times verglichen mit der "moral power which it may exert over the fortunes of our happy Union « eher »insignificant " gewesen. $^{\mathbf{1 9}}$

Zum zweiten hat die transfergeschichtliche Forschung bislang die Analyse von Austausch-
I 2 Zur Kristall-Metapher vgl. KLAUS Hammer, Bohemien, Astralhumorist, Katerpoetiker, Architekturphantast und Geschichten-Erfinder Paul Scheerbart - ein Utopist der Jahrhundertwende (2000) I-6.

I 3 Die folgende Analyse basiert auf der Auswertung der zeitgenössischen amerikanischen Berichterstattung sowie des Quellenmaterials in der Library of Congress, der New York Public Library und der New York Historical Society.

I4 Haltern, Die »Welt als Schaustellung « (I973a); MARTIN WÖRNER, Vergnügung und Belehrung. Volkskultur auf den Weltausstellungen I 8 5 I-I900 (I999). Gleiches gilt für Ilja Mieck und
C. T. Geppert, London vs. Paris (2004); ILJa Mieck, Deutschland und die Pariser Weltausstellung (I998).

I 5 Dan B. Fleming, JR., A West Virginia Boy at the New York World's Fair (2004) Io; Charles Hirschfeld, America on Exhibition (I957) IOI-I I 6; LINDA Hyman, Crystal Palace (I974); Robert C. Post, Reflections of American Science and Technology at the New York Crystal Palace Exhibition of I853 (I983) 337356; Ivan D. Steen, America's First World's Fair (1963) 256280.

I 6 IVAn Steen, New York I 853 (I999) I2.
I7 P. T. BARnum, The life of P. T. Barnum (I 855 ); DERS., P. T. Barnum and George S. Bryan, Struggles and triumphs: or, The life of P. T. Barnum (I927); J. BRYAN, The world's greatest showman: the life of P. T. Barnum (I956); Benjamin Jr. Silliman u. C. R. GOODRICH, The World of Science Art and Industry Illustrated from Examples in the New-York Exhibition I 853-I 854 (i 854 ); Georg Carstensen u. Karl Gildemeister, New York Crystal Palace (I 854).

I 8 New York Daily Times, I7. Juli I $852,2$.

I9 New York Times, I8. Juni I 853 . 
prozessen zwischen der Alten und der Neuen Welt ausgeklammert, wenngleich unter einer eher beziehungsgeschichtlichen Perspektive eine Reihe von Arbeiten vorliegt, die durchaus in diesen Kontext einzuordnen wären. ${ }^{20}$ Empirisch gründete die programmatische Entwicklung der transfergeschichtlichen Perspektive in der Analyse deutsch-französischer Austauschprozesse. ${ }^{2 \mathrm{I}}$ Mit den Arbeiten von Johannes Paulmann und Willibald Steinmetz wurde diese um den deutschbritischen Kontext ergänzt. ${ }^{22}$ Beeinflusst durch die Rezeption post-kolonialer Ansätze und die Debatte um Globalgeschichte entwickelte sich der Transferansatz weiter zu einem Untersuchungsinstrument für die vielfältigen Austauschprozesse zwischen Europa und der »nicht-westlichen « Welt. ${ }^{23}$ Dabei musste sich auch die Transferforschung mit der Kritik der postcolonial studies auseinandersetzen, dass ihr im Grunde ein unhintergehbarer Eurozentrismus innewohne. ${ }^{24}$ Möglicherweise ist es genau diese eurozentrische Perspektive, die der Betrachtung der Geschichte der USA, Kanadas, Australiens und Neuseelands als räumliche Erweiterung der europäischen Sozial-, Wirtschafts- und Kulturgeschichte Vorschub leistete. Zusammen mit Australien und Neuseeland gelten die USA und Kanada als »neo-europäische" Gesellschaften, die nicht nur durch europäische Siedler erschlossen wurden, sondern die sich im Sinne des französischen Konzeptes des Territoire d'Outre Mer als europäische Außenposten jenseits des Atlantiks entwickelt hätten. Europäische Ideen und europäische Institutionen seien die Grundlage für die gesellschaftlichen und politischen Modernisierungsprozesse, die diese Gesellschaften im I9. und frühen 20. Jahrhundert durchliefen.
Mit dem folgenden Beitrag soll das Bewusstsein für die Spezifik europäisch-amerikanischer Transferprozesse im I9. Jahrhundert, die in einem Kontext regionaler historischer Eigengesetzlichkeiten und spezifischer Entwicklungspfade stattfanden, geschärft werden. Die sozialhistorisch zentralen Prozesse des I8. und I9. Jahrhunderts verliefen nicht nur in den USA häufig historisch asynchron zu den entsprechenden Entwicklungen in Europa. Bereits während der Kolonialzeit entwickelten sich in Nordamerika aufgrund der recht unterschiedlichen lokalen Gegebenheiten politische, religiöse und kulturelle Formen, die als Ergebnis von kulturellen Transfers und Adaptionsprozessen verstanden werden müssen und nicht als einfache Übertragung europäischer Konzepte und Entwicklungen interpretiert werden dürfen. ${ }^{25}$ So ist die amerikanische Tradition des limited government, die im I9. Jahrhundert in den USA die Entwicklung des nationalstaatlichen Prinzips im europäischen Sinne bremste, ebenso als Abwehr autoritärer und zentralistischer Ausprägungen moderner Staatlichkeit wie als Zurückweisen des aristokratischen Prinzips, das die Entstehung des modernen Staates in Europa begleitet hatte, zu verstehen. Demokratie und Republikanismus waren Instrumente des Volkes zur Eindämmung des »Staates «. Die Zurückweisung des europäischen Staatsprinzips hatte jedenfalls im Hinblick auf die zentralen historischen Prozesse des I9. Jahrhunderts - Revolution und Staatsgründung, Westexpansion, Masseneinwanderung, Industrialisierung und Urbanisierung - einen enormen Einfluss auf die Herausbildung eigener Traditionen und spezifischer Strukturprobleme. Diese gilt es mit Hilfe des Transferansatzes zu erschließen. Publikationen mit so programmatischen
$20 \mathrm{Vgl}$. hierzu insbesondere die mittlerweile recht umfangreiche Literatur zur europäischen Einwanderung und zur Integration und Akkulturation der europäischen Einwanderer. Für einen Überblick zum Forschungsstand vgl. DIRK Hoerder, Cultures in contact (2002).

2 I Michel Espagne, Kulturtransfer und Fachgeschichte der Geisteswissenschaft (2000) 42-6I; DERS., Les tranferts culturels franco-allemands (I999).
22 Johannes Paulmann, Interkultureller Transfer zwischen Deutschland und Großbritannien (I998) 2I-43; Johannes Paulmann, Internationaler Vergleich und interkultureller Transfer (I998) 649-685.

23 Jürgen Osterhammel, Transferanalyse und Vergleich im Fernverhältnis (2003) 439-468; Sebastian Conrad u. Jürgen Osterhammel, Das Kaiserreich transnational (2004); JÜRGEN Osterhammel, Geschichtswis- senschaft jenseits des Nationalstaats (200I).

24 Sebastian Conrad u. Shalini RANDERIA, Geteilte Geschichten Europa in einer postkolonialen Welt (2002) 9-49.

$25 \mathrm{Vgl}$. hierzu das Forschungsprojekt "Colonial Governance und Mikrotechniken der Macht in englischen und französischen Kolonialbesitzungen Nordamerikas, I680I760« im Rahmen des SFB 700 »Governance in Räumen begrenzter Staatlichkeit«. 
Titeln wie »Der fremde Freund ${ }^{26}$ weisen auf das eigentümliche Spannungsverhältnis von Nähe und Fremdheit hin, das die Beziehungen zwischen Europa und Amerika prägt. Der transfergeschichtliche Ansatz, der unser Augenmerk auf genau die Aneignungs- und Abwehrprozesse lenkt, die zu den »feinen Unterschieden " (Bourdieu) in den europäischen und amerikanischen Gesellschaftssystemen geführt haben, hilft uns, Gemeinsamkeiten und Unterschiede - auch im Sinne der Akzeptanz von Eigenentwicklung historisch einzubetten, und sorgt damit nicht zuletzt auch für ein besseres Verständnis gegenwärtiger Spannungslagen.

\section{Imagining the Nation: \\ Die Weltausstellung von I 853 als \\ Instrument nationaler Prestigepolitik}

Die Forschungsliteratur zu Weltausstellungen im I9. und frühen 20. Jahrhundert weist fast stereotyp auf ihre Bedeutung als Projektionsfläche für den sich entwickelnden Nationalstaat und für die Visualisierung der Idee des Nationalen hin. Wie oben bereits erwähnt, waren Weltausstellungen letztlich »exercises in the imagery of nationalism «. ${ }^{27}$ Die Weltausstellungseuphorie verläuft zeitlich parallel zur Entwicklung und Etablierung des modernen, territorial definierten Nationalstaats und damit zu einem historischen Prozess, der nach Langewiesche zu den »erstaunlichsten Entwicklungen [gehört], die seit dem späten I 8. Jahrhundert Politik und Gesellschaft überall fundamental umgestalten $\ll{ }^{28}$ Über die
Visualisierung und Präsentation des Nationalen hinausgehend, dienten Weltausstellungen gegen Ende des I9. Jahrhunderts immer mehr auch der Ausstellung des Fremden, das zugleich im Zuge der Entstehung und Konsolidierung großer Überseeimperien zum Eigenen wurde. ${ }^{29}$ "Visions of Empire " sei - so Robert Rydell - der dominante Tenor amerikanischer Weltausstellungen seit I 876 gewesen. ${ }^{30}$ Weltausstellungen reflektieren damit auch den bekannten Zusammenhang von Nationalismus und Imperialismus.

Die Kristallpalastausstellung I85 I in London war der Auftakt zu einer in immer kürzerer Abfolge stattfindenden Veranstaltungsreihe, ${ }^{3 \mathrm{I}}$ die nicht allein der Präsentation des technischen Fortschritts gewidmet war, sondern vor allem auch der Darstellung des Wertebewusstseins der bürgerlichen Klasse. (Wirtschafts-)Nationalismus, der nicht an den territorialen Grenzen des eigenen Staates Halt machte, sondern im Rahmen des Kolonialismus zu einem Instrument der Universalisierung westlicher Werte wurde, gehörte zum ideellen Rahmen der Industrialisierung und diente der Legitimierung des Aufbaus eines den westlichen Kapitalismus fördernden internationalen Handelssystems. ${ }^{32}$ Als Medium und Instrument des friedlichen Wettbewerbs zwischen den Nationen versuchte jede Ausstellung größer, sensationeller und spektakulärer als die vorherige zu sein und entwickelte dabei eine spezifische Ausstellungskultur, die sich signifikant in der Ausstellungsarchitektur und der Entwicklung einer modernen Stadtplanung niederschlug (vgl. unten 2.).
26 Wolfgang Bergsdorf et al., Amerika, fremder Freund (2003); Klaus Harpprecht, Der fremde Freund (I982).

27 WÖRnER, Die Welt an einem Ort (2000) 4; vgl. auch Haltern, Die »Welt als Schaustellung « (I973a); BENEDICT, International exhibitions and national identity (I99I).

28 LANGEWIEsche, Nation, Nationalismus, Nationalstaat in Deutschland und Europa (2000) 42.

29 Peter H. Hoffenberg, An empire on display (200I).

30 Robert Rydell, Visions of Empire (I983) 37.

3 I I 853 New York, I 855 Paris, I 862 London, I 867 Paris, I873 Wien,
I 876 Philadelphia, I 878 , I 889 und I 890 abermals Paris, I 884 New Orleans, I 888 Glasgow, I 893 Chicago, I894 San Francisco, I895 Atlanta, I 898 Omaha (Nebraska), I90 I Buffalo (NY), I9OI-O2 Charleston (South Carolina), I904 St. Louis (Missouri), I905 Portland (Oregon), I907 Jamestown (Virginia), I909 Seattle (Washington), I9I 5 San Francisco, I9I 5-I6 San Diego

(California). Der Erste Weltkrieg und die Folgen führten in den I920er Jahren zunächst zu einer Pause im internationalen Ausstellungswesen. In den I93oer Jahren gewann die Idee jedoch wieder an
Momentum und trotz der großen Depression beteiligten sich auch die USA mit Ausstellungen in Chicago (I933-34), Dallas (I937), New York (I939-40) und San Francisco (I939-40) wieder an den internationalen Projekten.

32 Vgl. hierzu die Debatten um den Freihandel, die im Kontext von Weltausstellungen immer wieder politisch aktiviert wurden. Vgl. KAISER, Die Welt im Dorf (2000); Ursula Lehmkuhl, Creating Anglo-American Friendship (2005) 27-5I. 
Der universalistische und kosmopolitische Charakter von Weltausstellungen stieß in den USA im Grunde auf einen sehr empfänglichen ideellen Resonanzboden. Angesichts der nicht vorhandenen "gemeinsamen " Geschichte oder ethnonational zu begründender Traditionen basierte das amerikanische Selbstverständnis auf universalistischen Ideen und Idealen. Der universalistische und kosmopolitische Anspruch der Weltausstellung bediente die ideelle Basis der jungen amerikanischen Demokratie wie kaum ein anderes Medium des I9. Jahrhunderts. Weltausstellungen schienen geradezu ein Symbol des Americanism zu sein. Mit der Weltausstellung von I 853 hätten die Amerikaner - so Hirschfeld - ein Bild von sich selbst geschaffen. »The Crystal Palace was truly a symbol of the country, with all its promises and failures, its glories and meannesses. «33

»Like America, like its great poet Whitman, it [die Weltausstellung, U. L.] was sa divided, multiple personality`, compounded of boundless hopes and dark fears, of daring and caution, of boasts never quite realized. The Exhibition flung a challenge to aristocratic Europe even as it deferred to Old World tradition. It bespoke a radical utopian vision as well as hard-driven deals, a universal human fellowship and the petty calculations of merchants. It stood for the union of the states in face of the issues that were tearing it apart. It was American >Reaching outward «, slowly turning from its dream of isolated splendour in the garden continent of the west. « 34

Und doch kommt Robert Rydell in seiner Analyse der New Yorker Weltausstellung zu dem unausweichlichen Schluss: "As an effort to inspire Americans with a sense of nationalism, the
New York Crystal Palace Exhibition failed. «35 Wenn wir uns den historischen Kontext sowie die Umstände der Organisation der Ausstellung, die beteiligten Akteure und auch die Ausstellungsgegenstände insbesondere im symbolträchtigen Bereich von 'Kultur ‘ anschauen, wird schnell deutlich warum.

In Europa wurde das Bekenntnis zur Nation seit dem ausgehenden I 8. Jahrhundert mit Hilfe einer national orientierten symbolischen Politik oder über die politisch motivierte Förderung einer nationalen Erinnerungs- und Gedenkkultur in den "Lebenswelten, den Leitbildern und Verhaltensnormen der Menschen selber ${ }^{36}{ }^{6}$ verankert. ${ }^{37}$ Der Prozess der Nationsbildung wurde so von der Nationalisierung von Werten und Normen begleitet. Diese für die europäische Geschichte typische symbolische und lebensweltliche Verankerung der Idee der Nation fehlte im amerikanischen Kontext. Nach Revolution und Staatsgründung blieb die relative Unabhängigkeit der Einzelstaaten oberstes politisches Ziel. Die Form und Reichweite des amerikanischen Föderalismus bestimmte den politischen Diskurs nicht nur der Gründerväter, sondern auch der nachfolgenden Politikergeneration der frühen Republik. Die mit der Verfassung von 1787 gegründete Republik verstand sich als »Union «, als "a compact of many independent powers " (Jefferson 1825$)^{3^{8}}$ und nicht als "Nation «. Eine "Nation « im ethnonationalen Sinne, verstanden als Abstammungsgemeinschaft mit historischem Territorium und demokratischer Verfassungsordnung als Kernelementen, existierte Mitte des I9. Jahrhunderts in den USA nicht. ${ }^{39}$

Erst nach dem Bürgerkrieg setzte ein vorsichtiger, ja fast verhaltener Prozess der Natio-
33 Hirschfeld, America on Exhibition (I957) IOI.

34 Ibid., IOI.

35 Robert W. Rydell, John E. Findling u. Kimberly D. Pelle, Fair America: World's Fairs in the United States (2000) I7.

36 LANGewiesche, Nation, Nationalismus, Nationalstaat (2000) 42. Vgl. auch Pierre NorA, Les lieux de mémoire (I984); Etienne François u. Hagen SCHulze, Deutsche Erinnerungsorte (200I).
37 Zum Komplex »symbolische Politik «, » figurative Politik « oder "performative Politik « vgl. das Forschungsprogramm des SFB 584: "Das Politische als Kommunikationsraum in der Geschichte " sowie Ute Frevert u. HeinzGerhard Haupt, Neue Politikgeschichte (2005).

38 Jefferson, Letter to Edward Livingston, March 25, I825, in: Thomas JefFerson (I984) I6, II3.

39 Vgl. hierzu die von Anthony D. Smith zur Charakterisierung der
"Nation « vorgestellten Kriterien: I. ein historisches Territorium, 2. gemeinsame Mythen und historische Erinnerungen, 3. eine gemeinsame Massenkultur, 4. gemeinsame gesetzliche Rechte und Pflichten für alle Mitglieder der Nation, 5. eine gemeinsame Ökonomie mit territorialer Freizügigkeit für alle Angehörigen der Nation. Anthony D. Smith, The ethnic origins of nations (I987); ANThony D. SMITH, Nationalism (200I); DERS., Theories of nationalism (I983). 
nalstaatsbildung ein, ohne dass der 'Staat im amerikanischen politischen System jemals die institutionelle Macht und die politischen Steuerungskompetenzen seiner europäischen Äquivalente erreichte. Auch im gesellschaftlichen Bewusstsein spielt der `Staat allenfalls als negative Projektionsfläche für Warnungen vor politischem Machtmissbrauch und der Einschränkung individueller Freiheitsrechte eine Rolle. In vielerlei Hinsicht waren und blieben die USA eine Nation ohne Nationalstaat. Lebenswelten, Leitbilder und Verhaltensnormen waren im Jahrhundert der Masseneinwanderung nur in bedingtem Maße an das Bekenntnis zur Nation rückgebunden. Wichtiger waren die religiösen und politischen Gründungsmythen, die im Kontext des amerikanischen Puritanismus formuliert worden waren und sich in Kombination mit den demokratischen Prinzipien zur viel beschriebenen civil religion als ideologische Grundlage der jungen Republik verdichteten. So überrascht es nicht, dass die erste nativistische Bewegung, die in den I 85 oer Jahren in den USA aufkam und die man als negativen Ausdruck eines sich entwickelnden Nationalbewusstseins nennen kann, eine religiöse Stoßrichtung hatte. Sie richtete sich gegen den Katholizismus und die ihn repräsentierenden Einwanderergruppen, die Iren und die Deutschen. ${ }^{4 \circ}$

Die Union war in religiöser, ethnischer, wirtschaftlicher und sozialer Hinsicht ein äußerst heterogenes Konstrukt. Politisch war sie gespalten. Der Süden hielt weiterhin an der Sklaverei als Grundlage der Plantagenwirtschaft fest, u. a. um die mit der Industrialisierung Englands einhergehende gesteigerte Nachfrage nach Baumwolle in Europa befriedigen zu können. Unabhängigkeitskrieg und Revolution hatten dazu beigetragen, die demographischen und ideologischen Unterschiede zu verschärfen, die hinsicht- lich der Sklaverei von jeher zwischen Norden und Süden bestanden. Nach I 820 mit der nun forciert einsetzenden Erschließung des amerikanischen Westens bemühten sich die Gegner, das weitere Vordringen der peculiar institution in die Westgebiete zu verhindern. Die Probleme, die sich aus dem Zusammenhang von territorialer Expansion und Sklaverei für den Bestand der Union ergaben, traten seit den I840er Jahren immer deutlicher zu Tage. ${ }^{4 \mathrm{I}}$

Gleichzeitig setzte jedoch nach I 8 I 5 mit der Demokratisierung, der territorialen Expansion und der Verbreitung marktwirtschaftlicher Prinzipien ein Transformationsprozess ein, dessen wirtschaftliche und gesellschaftliche Dynamik kaum überschätzt werden kann. Die Entstehung eines nationalen Marktes und der Übergang vom Agrar- und Handelskapitalismus zur Industrialisierung gingen einher mit der Etablierung eines auf das private Interesse ausgerichteten Individualismus, der zusammen mit dem durch ihn entfesselten Wettbewerb zum Kern einer neuen, "liberalen " amerikanischen Identität wurde. Ökonomisches Wachstum und technische Neuerungen resultierten in tiefgreifenden Veränderungen im Denken und in den sozialen Beziehungen, und sie vergrößerten zudem die wirtschaftlichen und kulturellen Unterschiede zwischen den Nord- und Südstaaten. Die paradoxe Folge war, dass die Einbindung immer weiterer Bevölkerungskreise in eine nationale Marktwirtschaft die sozialen und regionalen Gegensätze verschärfte und damit die Gefahr des Zerfalls der Union vergrößerte. ${ }^{42}$

Insbesondere die traditionelle Ostküstenelite der Großhändler und Kaufleute und die sich um die Mitte des I9. Jahrhunderts sozial etablierende und an wirtschaftlicher und politischer Bedeutung gewinnende neue Schicht der Fabrikanten hatten die Notwendigkeit erkannt, zur

\footnotetext{
40 Vgl. David Harry Bennett, The party of fear (I995); JoHN HiGHAM, Strangers in the land (2002); Dale T. Knobel, America for the Americans (1996).

4I Vgl. Michael Burgan, A changing nation (2006); GEORGE D. HARMon, Aspects of slavery and expansion (I929).

42 Vgl. Donald R. Davis, The home market, trade, and industrial structure (I997), available from

http://www.ny.frb.org/research/ staffreports/sr 35 .html, RoNALD E. Seavoy, An economic history of the United States (2006). 
Sicherung des eigenen wirtschaftlichen Wohls der zunehmenden sozialen, politischen und wirtschaftlichen Spaltung der Nation entgegenzuwirken. Die amerikanische Beteiligung an der Weltausstellung in London hatte gezeigt, dass die Vereinigten Staaten ganz offensichtlich nicht nur »moralisch « im Sinne der civil religion den europäischen Nationen mit ihren aristokratischen Traditionen überlegen waren, sondern in vielerlei Hinsicht auch technologisch. Die Demonstration der »American superiority « 43 im Rahmen einer Weltausstellung in der Neuen Welt sollte deshalb als einigendes Band die vorhandenen politischen Gräben überspannen und zur Zementierung der Einheit der Nation beitragen. So jedenfalls beschrieben die Organisatoren der Ausstellung die politischen Ziele, die sie mit der Durchführung einer Weltausstellung in den USA verbanden. ${ }^{44}$ Von politischer Seite erhielten sie dabei jedoch keine Unterstützung. Wie zuvor bei der Organisation der amerikanischen Beteiligung an der Weltausstellung in London hielt sich die amerikanische Bundesregierung zurück. Die Organisation und Durchführung der Weltausstellung in New York war das Werk privater Akteure, deren primäres Motiv natürlich nicht politisch, sondern kommerziell war.

Die New Yorker Weltausstellung war das ideelle Kind eines Geschäftsmannes aus Boston, Edward Riddle, der als amerikanischer Commissioner die Weltausstellung in London begleitet und den Transport der amerikanischen Ausstellungsgegenstände organisiert hatte. Beeindruckt von dem enormen wirtschaftlichen Erfolg der Londoner Ausstellung - sie hatte einen Gewinn von I 50000 Pfund erwirtschaftet - wollte er eine vergleichbare Veranstaltung in New York durchführen und damit an den kommerziellen und den Publikumserfolg der Londoner Ausstellung, die
I 85 I mehr als 6 Millionen Besucher anlockte, anknüpfen. Es gelang ihm, eine politisch und wirtschaftlich recht einflussreiche Gruppe gleichgesinnter Geschäftsleute und Bankiers zur Finanzierung und Durchführung der ersten Weltausstellung auf amerikanischem Boden zusammenzustellen, die sich in der Association for the Exhibition of the Industry of All Nations, einer vom Staat New York zugelassenen privaten Handelsgesellschaft, zusammenschlossen. ${ }^{45}$ Wie sich jedoch recht bald herausstellte, konnte eine Weltausstellung nicht ganz ohne staatliche Hilfe durchgeführt werden. Vom Konzept her waren Weltausstellungen öffentliche Veranstaltungen, die nur mit finanzieller und materieller, aber auch mit der notwendigen symbolischen Unterstützung von Seiten der Politik erfolgreich sein konnten.

Zwar gelang es den Organisatoren, eine gewisse politische Unterstützung sicherzustellen. So erklärte das Finanzministerium das Ausstellungsgebäude zum Zollspeicher, um die Einfuhr der Ausstellungsgegenstände zu erleichtern. ${ }^{46}$ Der Secretary of State Webster verschickte Rundbriefe an die amerikanischen Konsulate, in denen er die offiziellen Vertreter der USA im Ausland aufforderte, die Requirierung und Einwerbung von ausländischen Ausstellungsteilnehmern mit allen Kräften zu unterstützen. ${ }^{47}$ Weitere finanzielle oder materielle Unterstützung aus Washington gab es jedoch nicht, wenngleich die offizielle Eröffnung der Ausstellung durch den Präsidenten als Ausdruck einer breiten politischen Unterstützung öffentlich vermarktet wurde. Angesichts der sich verschärfenden sektionalen Krisen misstraute die Bundesregierung den von den Organisatoren betonten »nation-building «-Potentialen der geplanten Ausstellung. Die politische Seite wurde nicht müde, den privaten
43 Rodgers, American Superiority at the World's Fair (I 852 ).

44 Vgl. hierzu auch die Berichterstattung in der New York Times, die nicht müde wurde auf das doppelte Ziel der Weltausstellung hinzuweisen: »Our advances in the study of nature should stand forth in the niches of the temple and embody the progress of the American mind. Let the great West and the great South roll their voices along the Palace aisles, and tell the world what stuff they are made of and what strides they have taken in the arts of business of life ... the impulse which this exhibition will give to the mechanical and artistic glories of this country is insignificant when compared with the moral power which it may exert over the fortunes of our happy union. "New York Times, I8. Juni 1853 .

$45 \mathrm{Zu}$ den Mitgliedern der Association gehörten August Belmont,
Alexander Hamilton, Jr., William Cullen Bryant, Edward K. Collins und Mitglieder der Familien Schuylers und der Livingstons. Somit bestand die Association aus New Yorkern, die einen festen Hintergrund und enge Beziehungen zur Wall Street, nach Washington und London hatten.

46 Steen, America's First World's Fair (I963) I2-I3.

47 Hirschfeld, America on Exhibition (I957) IO4. 
Charakter des Unternehmens zu betonen, wohl auch, um sich von den Fehlplanungen zu distanzieren, die von Seiten der europäischen Teilnehmer öffentlich kritisiert wurden. ${ }^{48}$ Damit verkannte die amerikanische Bundesregierung die große politische Chance, die die Weltausstellung einerseits als Instrument der Machtprojektion nach außen, andererseits für die Identitätsbildungsprozesse im Innern bot. Ein mangelndes nationalstaatliches Bewusstsein und die Tradition des limited government, die sich u.a. in der sich zu dieser Zeit fast ausschließlich auf den Eisenbahnbau konzentrierenden wirtschaftlichen Entwicklungspolitik und dem Fehlen einer wirtschaftspolitischen Gesamtstrategie niederschlugen, trugen nicht unerheblich zum Scheitern des Experiments , Weltausstellung $<$ bei. ${ }^{49}$

Die Institution /Weltausstellung traf Mitte des I9. Jahrhunderts in den USA auf wirtschaftliche und politische Rahmenbedingungen, die eine Umsetzung im Sinne der europäischen Tradition erschwerten, wenn nicht gar unmöglich machten. Zunächst ist dabei die fehlende lebensweltliche Verankerung der nationalen Idee als eine der Ursachen für das Scheitern zu nennen. Obwohl ideengeschichtlich die zentralen Konzepte der Weltausstellung - Universalismus und Kosmopolitismus - in der amerikanischen Tradition einen idealen Resonanzboden fanden, konnte das Konzept >Weltausstellung< I 853 nicht als Instrument nationaler Politik fungieren. Es fehlte dazu nicht nur das Interesse von Seiten der Bundesregierung, sondern vor allem auch der ideelle Rahmen - die Idee der Nation -, die die europäischen Nationalstaaten zum friedlichen Wettbewerb anspornte und Weltausstellungen als Medium nationaler Prestigepolitik wirksam werden ließ. Darüber hinaus waren die USA um I 850 - im Unterschied zu Großbritannien und in gewisser Weise auch zu Frankreich - noch eine vornehmlich agrarisch strukturierte Gesellschaft. Der Industrialisierungsprozess hatte noch nicht in einem mit Europa vergleichbaren Maße eingesetzt. Dies führte, zusammen mit der politischen Spaltung der Nation, dazu, dass auch die einsetzende wirtschaftsnationale Dynamik politisch nicht genutzt wurde. Die Weltausstellung von I 853 markiert allerdings den historischen Moment, in dem sich Wissenschaft und Technologie von einem eher sekundären zum zentralen Schlüssel der amerikanischen Geschichte im I9. Jahrhundert entwickelten. ${ }^{\circ}$

\section{The World in the City: \\ Weltausstellungen \\ als globale öffentliche Räume}

Der fehlende snationale< Kontext hatte unmittelbare Auswirkungen auf die Übertragung der zweiten zentralen Funktion von Weltausstellungen: die Schaffung öffentlicher Räume, die ein Forum für die unterschiedlichen Vertreter aus den Bereichen Politik, Wirtschaft, Wissenschaft, Technik, Bildung, Kunst und Unterhaltung boten - ein Forum, das im 19. Jahrhundert für die Netzwerkbildung und die gemeinsame Diskussion von Zukunftsstrategien zentral war. Öffentliche Räume im Sinne der griechischen agora stellen ein zentrales Element städtischen Lebens dar. Die Entstehung globaler öffentlicher Räume im I9. Jahrhundert verlief räumlich und zeitlich parallel zur Entwicklung von Metropolen, die als Laboratorium des beginnenden Globalisierungsprozesses fungierten. Weltausstellungen brachten die Welt an einem Ort zusammen und boten einen Kommunikationsraum für entstehende transnationale Netzwerke.

New York war in der Mitte des I9. Jahrhunderts ein Ort, der die Heterogenität, aber auch die enorme Entwicklungsdynamik der Ver-

\footnotetext{
48 Zur Kritik an der Planung des Baus sowie der Organisation der Ausstellung selbst vgl. insbesondere die Berichterstattung im Scientific American, z. B. in der Nummer 38 v. 4. Juni 1853 . 49 Vgl. Andreas Etges, Wirtschaftsnationalismus (I999). 50 Dies lässt sich sehr schön an der Anzahl der zugelassenen Patente belegen. I 853 betrug die Gesamtzahl der Patente in der jungen
}

Republik 20000. 23 Jahre später, während der Centennial Exposition in Philadelphia, hatte sich die Anzahl verzehnfacht und betrug 200000. Der Commissioner of Patents unterzeichnete während der Centennial Exposition routinemäßig über rooo Patente pro Monat. Vier Jahrzehnte nach der New Yorker Weltausstellung, I 893 während der Chicagoer Weltausstellung, wurden mehr
Patente in einem einzigen Jahr ausgegeben, als 1853 insgesamt vorhanden waren. Vgl. ROBERT C. Post, 'Liberalizers` versus Scientific Men in the Antebellum Patent Office (I976) 24-54. 
einigten Staaten gleichsam in einem Mikrokosmos abbildete. Bereits I683, als die erste Stadturkunde für New York ausgestellt wurde, lebten auf der Insel Manhattan fast 4000 Menschen. Eine für die damalige Zeit in Nordamerika enorm große Zahl, die sich allerdings verglichen mit London, das zu Beginn des I 8. Jahrhunderts schon etwa 600000 Einwohner zählte, eher bescheiden ausnimmt. Bis zum Beginn des I9. Jahrhunderts war New York auf 35000 Einwohner angewachsen. $\mathrm{Zu}$ dieser Zeit war London mit über I,2 Millionen Einwohnern bereits die größte Stadt der Welt. Für beide Städte gilt, dass die erste Hälfte des I9. Jahrhunderts eine Periode beschleunigten Wachstums darstellte. Während London bis I 850 seine Einwohnerzahl verdoppelte, vergrößerte sich New York um das zehnfache auf 3 I 2000 Einwohner im Jahr $1840 .{ }^{51}$ Im Falle New Yorks hatten vor allem zwei zentrale Faktoren zu dieser rapiden Entwicklung der Stadt beigetragen: die Börsengründung in der Wall Street im Jahr I792, durch die New York zum amerikanischen Finanzzentrum aufstieg, und die Fertigstellung des Eriekanals I 825. Durch ihn wurde eine Verbindung zwischen New York, den Großen Seen und dem Mittleren Westen geschaffen. Über Nacht wurde die Stadt zum größten Warenumschlagsplatz an der amerikanischen Ostküste und damit zu einem wirtschaftlichen Konkurrenten für Boston, das bis dahin das Wirtschafts- und Handelszentrum darstellte. Bereits I 830 wurden fast 40 Prozent des internationalen Handels über New York abgewickelt. ${ }^{52}$

Die dynamische Entwicklung der Stadt in den folgenden 90 Jahren vorwegnehmend, begann in den I 8 Ioer Jahren eine systematische Stadtplanung. Nach einem I 8 I I von John Randall vorgelegten Plan wurde die gesamte Insel Manhattan, von der bis dahin nur die Südspitze bebaut war, mit einem Straßennetz überzogen, das Manhattan in ein Rechteckraster einteilte, bestehend aus 30 Meter breiten Avenues in nordsüdlicher Richtung und I 8 Meter breiten durchnummerierten Querstraßen in ost-westlicher Richtung. Die einzige Ausnahme war und ist bis heute der Broadway. John Randalls Plan sah bereits eine Bebauung bis zur I 55. Straße vor (vgl. Abb. I). Diese wird aber erst Ende des I9. Jahrhunderts erreicht. Erst dann, I898, wurden auch die heutigen Stadtteile Manhattan, Queens, Bronx und Staten Island zu Greater New York zusammengefasst. Mit seinen nunmehr 4,2 Millionen Einwohnern gehörte New York am Ende des I9. Jahrhunderts zusammen mit London und Paris zu den drei größten Städten der Welt. ${ }^{53}$

Bereits Anfang des I9. Jahrhunderts stand New York als Symbol für Wohlstand und außergewöhnlichen Reichtum. ${ }^{54}$ Die äußerst günstige Lage am Hafen förderte die Güterproduktion. Die Wohnbezirke der bürgerlichen Oberschicht, der American Bourgeoisie, ${ }^{55}$ die sich aus Kaufleuten, Bankiers, Fabrikanten, Notaren, Ärzten und anderen Freiberuflern zusammensetzte, breiteten sich in den Norden und in die Randbezirke aus. Ähnlich wie in London, das mit der Herausbildung von Westend und Eastend eine sozialgeographische Teilung der Stadt erlebte, entwickelte sich in der ersten Hälfte des I9. Jahrhunderts eine soziale Topographie, die den Charakter New Yorks bis Mitte des 20. Jahrhunderts bestimmen sollte. Während im Süden und Südosten der Stadt, insbesondere in der zwischen dem Chatham Square North und der E.4th Street gelegenen Bowery und dem angrenzenden Viertel Five Points, dort wo sich die Fabriken und die Hafenanlagen und damit die Wohnviertel der Arbeiter und Immigranten befanden, Armut, Elend und Gewalt herrschten, ${ }^{56}$ entstanden im
5 I Vgl. Felix Driver u. David Gilbert, Imperial cities (I999); Hermione Hobhouse, Thomas Cubitt: master builder (I97I); Celina Fox, London - World City, I800-I 840 (I992); AsA Briggs, Victorian Cities (I968). 52 Mona Domosh, Invented cities (I996) 7-34; vgl. auch CHARLES A. JonEs, International business in the nineteenth century (I987);
LARS Magnusson, Free trade: I793-I886 (I996).

53 Tertius Chandler, Four thousand years of urban growth (I987).

54 Sven Beckert hat in seiner Stadtgeschichte New Yorks, die den treffenden Titel »Monied Metropolis « trägt, auf der Grundlage einer aus dem Jahr I 855 stammenden Selbsteinschätzung von 324 New Yorkern, die über einen persönlichen Besitz von mehr als Io 000 Dollar verfügten, die Dominanz des Handels (40\%) und des herstellenden Gewerbes (20\%) herausgearbeitet. SvEn BECKERT, The Monied Metropolis (200I) 2 I.

55 Ibid.

56 Vgl. Clint Willis, Crimes of New York (2003). 
Nordwesten und den westlichen Randbezirken, in die sich die wohlhabende Schicht zunehmend zurückzog, die ersten Prachtbauten. Der Madison Square und die Fifth Avenue entwickelten sich in den I840er Jahren zu den bevorzugten Wohngebieten der American Bourgeoisie. Diese errichtete eine eigene, sozial exklusive Welt, mit Riten und symbolischen Handlungen, die der Netzwerkbildung und der Stabilisierung sozialer Beziehungen dienten und einen ausgesprochen selbstreferentiellen Charakter hatte. ${ }^{57}$ Die so entstandene, über >Kultur definierte Gruppe

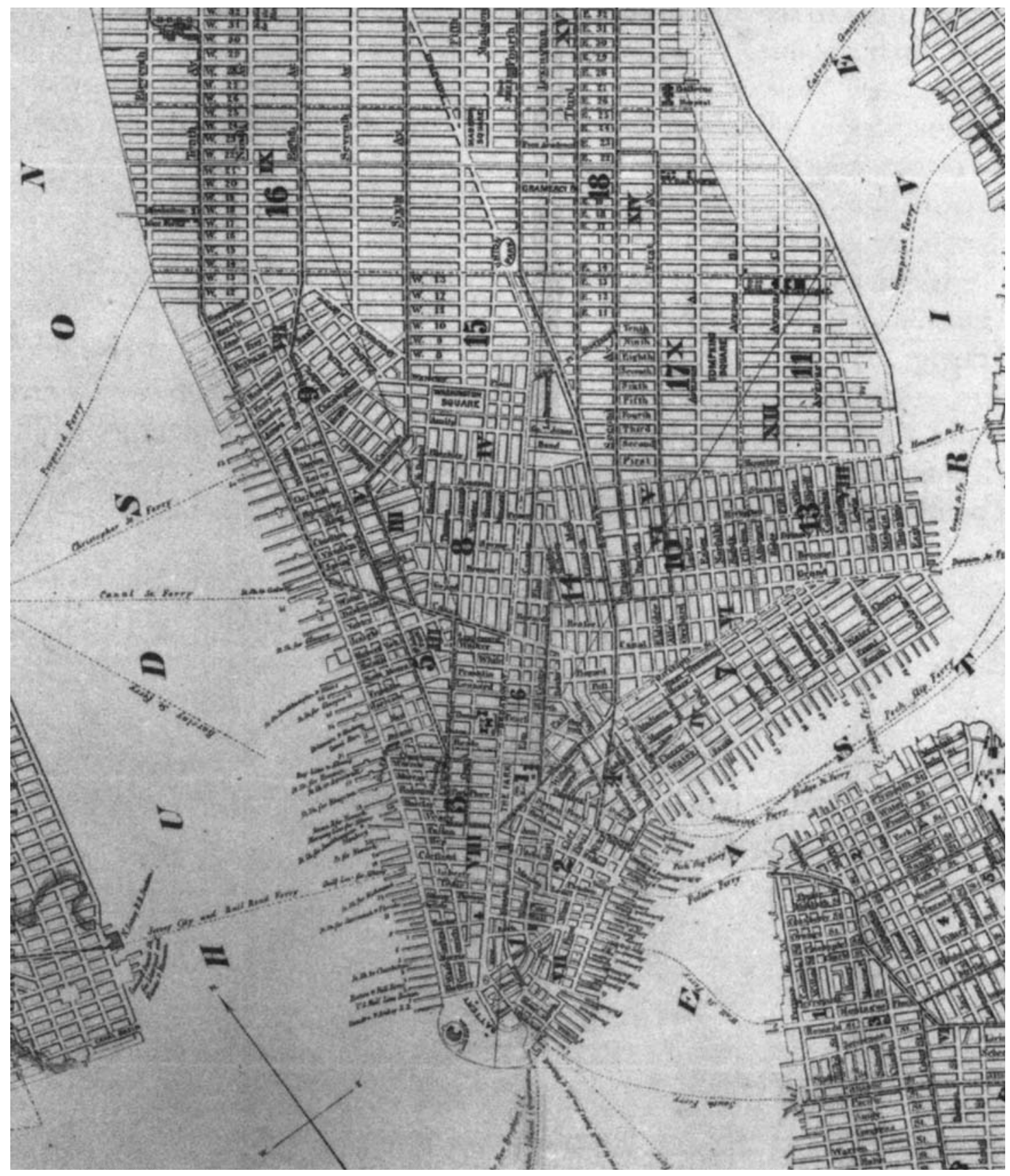

๕.

Abb. I: Map of New York City in I 859 .

Q: Harvard Map Collection. 
schirmte sich zunehmend von der Armut und dem Elend, die das städtische Leben außerhalb ihrer eigenen Welt bestimmten, ab.

Bereits zu Beginn des I9. Jahrhunderts lebte jeder siebte New Yorker in Slums. Aufgrund des Schmutzes und des Abfalls in den Straßen, fehlender Kanalisation, mangelhafter Trinkwasserversorgung und einer extremen Wohndichte breiteten sich Infektionskrankheiten wie Cholera, Typhus und Diphtherie enorm schnell aus. In New York gab es im 19. und frühen 20. Jahrhundert allein vier Choleraepidemien. I 849 starben dabei mehr als 5000 Einwohner. I88I und I 887 wurde die Stadt von Diphtherieepidemien heimgesucht, die eine ebenso große Zahl von Toten nach sich zog, während I 864 und I 865 Tausende an Typhus starben. Die unhygienischen Lebensbedingungen in New York bedingten auch die enorm hohe Säuglingssterblichkeit. I 840 starben ca. I90 von I 000 Neugeborenen im ersten Lebensjahr. ${ }^{58}$ Außerdem wurde New York in regelmäßigen Abständen von Großbränden heimgesucht, die teilweise ganze Viertel vernichteten. ${ }^{59}$

Doch nicht nur die scharf getrennten sozialen Welten und die große Armut kennzeichneten New York. Im Unterschied zu den europäischen Metropolen Paris und London fehlte der Stadt zur Jahrhundertmitte trotz ihrer Größe im Hinblick auf Bevölkerungszahl und im deutlichen Kontrast zu ihrem Reichtum immer noch ein zentrales Element städtischen Lebens: öffentliche Räume und kulturelle Einrichtungen. Da es keine öffentlich oder staatlich organisierte Förderung und Begleitung der Stadtentwicklung im europäischen Sinne gab, ${ }^{60}$ war die Einrichtung solcher Räume der privaten Initiative überlassen. Während in Großbritannien, Frankreich und Deutschland seit dem Ende des I8. Jahrhunderts staatlich geförderte Architektenwettbewerbe zum Bau von Museen, Theatern oder Opernhäusern und damit zur Ausbildung öffentlicher Räume beitrugen, ${ }^{6}{ }^{6}$ konzentrierte sich in den USA Stadtplanung noch auf die Grundvoraussetzungen von Stadtentwicklung wie das Anlegen von Straßen und Wohndistrikten. Die New Yorker Elite hatte ihrerseits kaum in öffentliche Räume investiert und kämpfte um die Kontrolle über die wenigen kulturellen Einrichtungen, die es gab. ${ }^{62}$ Erst im Verlauf der 185 oer Jahre entwickelte sich vom Madison Square ausgehend ein Theater- und Museumsdistrikt. ${ }^{63}$ Eine zentrale Rolle spielte dabei der ${ }^{8} 854$ begonnene und $\mathrm{I} 858$ vollendete Bau des berühmten Fifth Avenue Hotel. I 854 wurde auch die Academy of Music eröffnet, ein Opernhaus nach europäischem Vorbild an der Nordseite der I4. Strasse und östlich von Irving Place. ${ }^{64}$ Vier Jahre später entstand am Madison Square das Fifth Avenue Opera House, das sich in den I 86oer Jahren zum Ort der Massenunterhaltung entwickelte: Die populären Christy Minstrel Shows und leichte Komödien dominierten das Programm. ${ }^{65}$

In diesem Klima städtischer Entwicklung beantragte Edward Riddle zusammen mit seiner
57 Beckert, Monied Metropolis (200I) 45 .

58 Vgl. Evelynn Maxine HamMONDs, Childhood's Deadly Scourge (I999); Judith Walzer LeavitT, Typhoid Mary (I996); NaOmi Rogers, Dirt and Desease (I992).

59 Vgl. Stephen F. Ginsberg, The Police and Fire Protection in New York City (I97I) I33-I 50; Donald James Cannon, The Fire Department of the City of New York (I976).

60 In New York dominierte seit den I790er Jahren die Tammany Society, die als private Wohlfahrtsorganisation gegründet worden war und sich in der ersten Hälfte des I9. Jahrhunderts immer mehr zum politischen Arm der demokratischen Partei entwickelte. Als solcher prägte sie die New Yorker Kommunalpolitik maßgeblich. Vgl. Jerome Mushiat, The reconstruction of the New York democracy, I86I-I874 (I98I); DERS., Tammany: the evolution of a political machine, I789-I 865 (I97I); WARREN SLOAT, A battle for the soul of New York (2002).

6I Tony Bennett, The Exhibitionary Complex (I988) 8I.

62 Sven Beckert, Monied Metropolis (2002) 46.

63 M. Christine Boyer, Manhattan Manners (I985); ERIC HoMBERGER, The historical atlas of New York City (1994).
64 Boyer, Manhattan Manners (I985) 69.

65 Vgl. Lynn Аввотt u. Doug SeROFF, Ragged but right (2007); AnNemarie Bean, James Vernon Hatch u. Brooks McNamara, Inside the minstrel mask (I996); Sarah Meer, Uncle Tom mania (2005); Mark E. Neely, The boundaries of American political culture in the Civil War era (2005); Thomas Laurence RiIs u. a., Brookly College (I992); RoBert C. Toll, Blacking up: the minstrel show in nineteenth century America (I974); DAVID Van Veersbilck, u. a. The Early minstrel show (I985). 
Investorengruppe im Herbst $185 \mathrm{I}$ in einer Petition an das Board of Alderman der City of New York, " to erect on Madison Square a building of iron and glass, 600 feet long and 200 feet wide, for an Industrial Exhibition of all Nations «. ${ }^{\mathbf{6 6}}$ Der Ort war unter dem Gesichtspunkt der Stadtentwicklung klug gewählt, wenngleich ihm der Charme und die Weite, aber auch die symbolische Bedeutung, die Hyde Park Mitte des I9. Jahrhunderts besaß, noch fehlte. Zeitlich kam der Antrag aber mindestens drei Jahre zu früh. Im Herbst I85 I lag die große CholeraEpidemie gerade einmal zwei Jahre zurück. Die in der Regel durch eine public private partnership geförderte Entwicklung öffentlicher Kultureinrichtungen sollte erst drei Jahre später einsetzen. Riddle war insofern deutlich ein Mann vor seiner Zeit. Die öffentliche Reaktion auf seine Petition, die vom Board of Alderman zunächst positiv beschieden wurde, macht dies sehr deutlich.

Wie üblich wurden die Entscheidungen der Stadtverwaltung in der lokalen Presse veröffentlicht. Nach der Bekanntgabe der Bewilligung des Baus eines Glas-Eisen-Gebäudes am Madison Square regte sich, nicht zuletzt wegen der mangelnden öffentlichen Information über die geplante Weltausstellung, massiver Protest von Seiten der Anwohner. Durchaus vergleichbar mit den Kommentaren einiger konservativer Vertreter im britischen Oberhaus, wie etwa des konservativen MP Col. Charles de Laet Waldo Sibthorpe, der die Londoner Ausstellung als »the greatest trash, the greatest fraud, and the greatest imposition ever attempted to be palmed upon the people of this country « bezeichnete und davor warnte, dass sie die Pest nach London bringen könnte, ${ }^{67}$ fürchtete die am Madison Square wohnende New Yorker Elite, dass der geplante Kristallpalast die Ästhetik des Platzes beein- trächtigen könnte und dass der zu erwartende Verkehr zu einer erheblichen Belästigung der Anwohner führen werde. ${ }^{68}$

Während in London die Standortfrage mit der gesamten politischen Macht der Royal Commission und ihres Vorsitzenden Prinz Albert in der entscheidenden Debatte im britischen Parlament am 4. Juni I850 letztlich erfolgreich im Sinne der Royal Commission gelöst werden konnte, war es der privaten Association nicht möglich, ihren Vorschlag durchzusetzen, den Kristallpalast im damaligen kulturellen Herzen der Stadt, am Madison Square, zu errichten. Der Chief Justice von New York, der aufgrund der Anwohnerproteste die Petition erneut verhandelte, nahm letztlich die Entscheidung des Board of Alderman zurück und gestattete stattdessen die Nutzung des Reservoir Square an der 42. Straße, zwischen der Fifth Avenue und der heutigen Avenue of the Americas. ${ }^{69}$ Die Ausstellung war damit gezwungen an den noch kaum besiedelten Nordrand von Manhattan zu ziehen und das Weltausstellungsgebäude am Reservoir Square, dem heutigen Bryant Park und Standort der New York Public Library, zu errichten. Dies war in verschiedener Hinsicht eine problematische Entscheidung. Zunächst musste das geplante Bauwerk, das im Falle der Londoner Weltausstellung nicht unerheblich zur Attraktivität der Veranstaltung beigetragen hatte und sich selbst zu einem Publikumsmagneten entwickelte, im Reservoir Square mit dem massiven Bauwerk des Croton Reservoir, dem zentralen Trinkwasserreservoir New Yorks, konkurrieren (vgl. Abb. 2).

Das geplante Ausstellungsgebäude, das wie der Londoner Kristallpalast nicht nur einen globalen öffentlichen Raum konstituieren, sondern auch architektonisch ein Zeichen des Fortschritts setzen sollte, wurde damit räumlich marginali-

66 New York Times, 26. Dezember I $85 \mathrm{I}$.

67 Zit. n. http://www.learningcurve. gov.uk/victorianbritain/lawless/ [8.5.2006].

68 Vgl. New York Daily Times, 3I. Januar I852.

69 Reservoir Square wurde Anfang des 20. Jahrhunderts in Bryant Park umbenannt.

ஜั.

Ein Kristallpalast für New York 
siert und im Hinblick auf seine symbolischen Leistungen entwertet. Weitere Konkurrenz gab es durch die zeitgleich erfolgte Eröffnung des berühmten Hippodromes von Franconi - ein zweistöckiges Zirkuszelt, das Io००० Besucher fasste. Zwei Jahre lang, praktisch zeitlich parallel zur Weltausstellung, bot es ausgerechnet am Madison Square, dem von den Organisatoren der Weltausstellung präferierten Ort, Unterhaltung für ein Massenpublikum: Wagenrennen, tanzende Pferde und die berühmte Course des Singes, ein Ponyrennen mit Affen als Jockeys.

Trotz der ungünstigen Ausgangsbedingungen beteiligten sich 5272 Aussteller an der Weltausstellung, von denen etwa die Hälfte aus dem Ausland kamen. ${ }^{70}$ Verglichen mit den fast
I 4000 Ausstellern, die mehr als I00000 Ausstellungsstücke im Londoner Kristallpalast zur Schau gestellt hatten, ${ }^{7 \text { I }}$ wirkt die Beteiligung allerdings eher bescheiden. Wie in London wurden jene Ausstellungsstücke prämiert, die sich durch besondere Innovation und einen besonderen Beitrag zum technischen Fortschritt auszeichneten. Nicht zuletzt aufgrund der numerischen Dominanz amerikanischer Ausstellungsgegenstände schnitten auch diesmal die amerikanischen Aussteller wieder sehr gut ab. Amerikanische Firmen wie Singer, die mit ihren Nähmaschinen einen Publikumserfolg erzielten, oder Erfinder wie Samuel Morse, der seinen Telegraphen ausstellte, erhielten Auszeichnungen und Preise. ${ }^{72}$

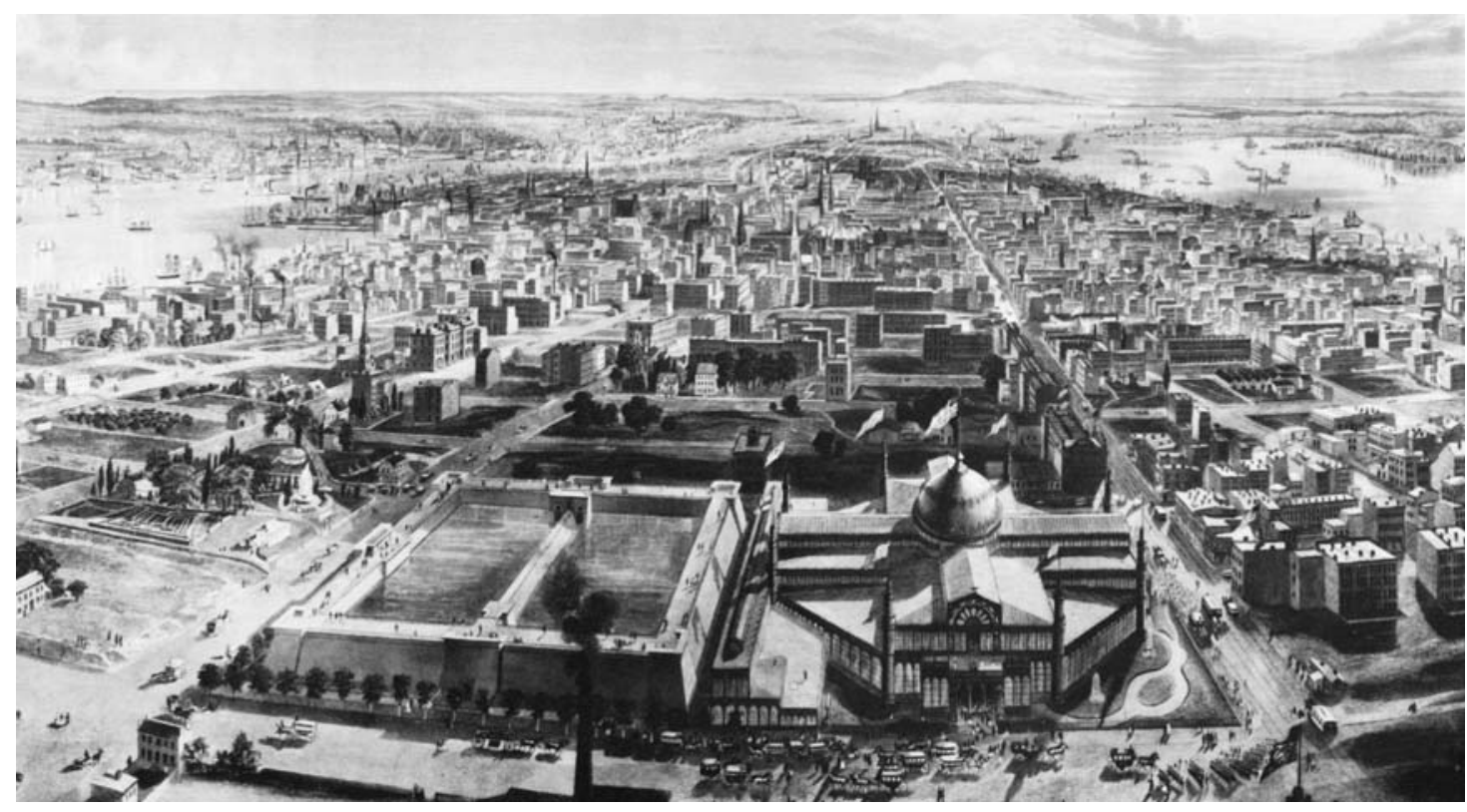

Abb. 2: Croton Reservoir und Kristallpalast.

Q: Central building, paintings, N.Y. in I 855 from Latting Tower by B. F. Smith: New York from the Latting Observatory, showing reservoir and Crystal Palace, New York Public Library.

70 Der Katalog zur Weltausstellung in New York listet I 467 amerikanische Aussteller, 58 I britische, 639 deutsche, 4I 8 französische, I06 Schweizer, Iо I holländische, 270 österreichische, I07 italienische und weitere 98 Aussteller aus den britischen Kolonien auf. Official catalogue of the New York exhibition of the industry of all nations, New York I 853 . 7i Hermione Hobhouse, The Crystal Palace and the Great Exhibition (2004) 66.

72 Für eine Beschreibung ausgewählter neuer Erfindungen vgl. Scientific American, I7. September I 853 ; The New England Farmer, September $1853,5,9$. 
Im Unterschied zur Londoner Ausstellung wurde in New York auch Kunst präsentiert. Es gab eine umfangreiche Ausstellung zeitgenössischer Malerei, die die Ausstellung von Skulpturen ergänzte und von den zeitgenössischen Beobachtern als zentraler Beitrag zur kulturellen Entwicklung der Vereinigten Staaten herausgestellt wurde. ${ }^{73}$ Darüber hinaus bot die Weltausstellung in den weitgehend leeren Seitenflügeln des Ausstellungsgebäudes side shows: Arenen für Hahnenkämpfe, Wettbuden, Getränkestände, sogenannte grog shops, die alkoholische Getränke anboten. Die Seitenflügel dienten darüber hinaus als Ausstellungsfläche für Kuriositäten und Exotismen: Zweiköpfige Kälber, tanzende Bären, Zwerge, Riesen, Klapperschlangen und was sonst noch zum Repertoire zeitgenössischer Kuriositätenkabinette gehörte, wurden hier präsentiert. Die populären Elemente der Weltausstellung irritierten den respectable citizen und standen in einem eigentümlichen Kontrast zu den Bildergalerien und Skulpturenausstellungen, mit denen die Organisatoren den zivilisatorischkulturellen Fortschritt der jungen Nation zur Schau stellen wollten. In Europa wurden Unterhaltungselemente für das Massenpublikum erst gegen Ende des I9. Jahrhunderts in die Weltausstellungen integriert, als auch Paris, London oder Wien auf die Bedürfnisse und Wünsche des Massenpublikums reagierten und ihren Besuchern ein Angebot aus »Vergnügung und Belehrung" offerierten. ${ }^{74}$

Trotz des breiten Unterhaltungsangebots entwickelte sich die Weltausstellung nicht, wie von den privaten Investoren erhofft, zu einem Publikumsmagneten. Der gewünschte wirtschaftliche Erfolg blieb aus. Schuld daran waren nicht nur die bereits genannten strukturellen Probleme, sondern auch ganz konkrete bauliche und organisatorische Mängel. Letztere hatten die
Organisatoren gezwungen, den für Mai I 853 geplanten Eröffnungstermin immer weiter in den Sommer hinein zu verschieben. Auch nach der Eröffnung wiesen die großzügig bemessenen Ausstellungshallen wochenlang große Lücken auf, weil noch nicht alle Ausstellungsstücke in New York angekommen und aufgestellt waren. Schließlich warnten lokale Zeitungen im Süden und Nordwesten der USA ihre Leser davor, voreilig nach New York zu fahren. Sie empfahlen zu warten, bis die Ausstellung komplettiert worden sei. So vergingen die Sommermonate und der erwartete Besucherstrom sowie die kalkulierten Einnahmen blieben aus. Weniger als ein Jahr nach der feierlichen Eröffnung am I4. Juli I 853 trat Thomas Sedgwick als Präsident der Weltausstellungsgesellschaft mit über I00 000 Dollar unbezahlter Rechnungen in der Tasche zurück. Die Association versuchte das Unternehmen wiederzubeleben, indem sie dem erfolgreichen Unternehmer und amerikanischen Zirkuspionier P.T. Barnum die Präsidentschaft in der Association anbot. ${ }^{75}$ Barnum verzichtete aber angesichts des überwältigenden Schuldenberges auf die ehrenvolle Aufgabe. Am I. November I 854 schloss die Weltausstellung mit einem Defizit von insgesamt 300000 Dollar. Das Gebäude blieb zunächst stehen und wurde zur Durchführung größerer Veranstaltungen vermietet, bis es schließlich im Oktober I 857, während der Jahresausstellung des American Institute, niederbrannte.

\section{The Crystal Palace:}

Ausstellungsarchitektur als Zeichen von Fortschritt und Modernität

Die Organisatoren der New Yorker Weltausstellung versuchten mit ihrem Ausstellungskonzept eine merkwürdige und aus europäischer

\footnotetext{
73 New York Daily Times, 30. Juni I 853 ; The Literary World, vol. I7. Dezember I8 53, I3, I9; Godey's Lady's Book, Vol. 47, Oktober I 853, 3778; Forrester's Boys' and Girls' Magazine and Fireside Companion, I. Oktober I 853 , I24.

74 Vgl. WÖRNER, Vergnügung und Belehrung (I999).

75 Vgl. Catherine M. Andronik, Prince of Humbug (I994); Alice
}

Mulcahey Fleming, P. T. Barnum (I993); Joe Vitale, There's a customer born every minute (2006). 
Perspektive sicherlich ungewöhnliche Quadratur des Kreises: Mit der Ausstellung und dem Ausstellungsgebäude sollte ein Symbol des Fortschritts und der Modernität nach New York geholt werden. Die Organisatoren knüpften dabei an symbolische Traditionen an, die einen großen Teil der Faszination des Kristallpalastes erklären. In der europäischen Romantik hatte der Kristall als Bild der Perfektion die Landschaftswahrnehmung und -beschreibung maßgeblich geprägt. Diese kristalline Tradition findet Eingang in die Architektur, insbesondere in die Glasarchitektur als Verkörperung des Utopischen. ${ }^{76}$ Sie wirkt damit als Brückenelement, mit dem romantische Vorstellungen in die Moderne hinübergerettet werden. Die Bezeichnung des Londoner Glashauses als »Kristallpalast « ist Ausdruck dieser Brückenfunktion des Kristallmotivs. Es versöhnt das Bekannte mit dem Neuen und verleiht dem Neuen zugleich ein flexibles Symbol für die Kommunikation seiner zentralen Pfeiler: Modernität und Fortschritt. Es ist diese kristalline Tradition der europäischen Romantik, die den Londoner Kristallpalast zu einem europäischen lieu de mémoire werden ließ. Eine solche kristalline Tradition fehlt in der amerikanischen Romantik. Das Motiv taucht erst im Gilded Age in den überladenen viktorianischen Innendekorationen auf, dann allerdings ohne den magischen und utopischen Bezug, in einer eher domestizierten Form. ${ }^{77}$ So trug nicht nur der Ort, an dem der New Yorker Kristallpalast gebaut wurde, zur symbolischen Entwertung der Ausstellungsarchitektur bei. Vielmehr erwies sich die Metapher im amerikanischen Kontext als dysfunktional, fehlte ihr doch der kulturelle Resonanzboden, der den geplanten Kristallpalast als Symbol für den Anbruch eines neuen Zeitalters ins kollektive Bewusstsein hätte heben können. Während in London bereits die
Architektur als Symbol für Modernisierung und Fortschritt stand und damit die für Weltausstellungen typische Symbiose und auch Dialektik von Innen und Außen, von Präsentation und Repräsentation verkörperte, verhielten sich die amerikanischen Organisatoren im Hinblick auf die Repräsentationsfunktion der gewählten Ausstellungsarchitektur eher konservativ. Zwar sollte ein Gebäude errichtet werden, das in Größe und im Hinblick auf die architektonische Leistung und Modernität dem Londoner Kristallpalast gleich kam. Dennoch entschied man sich für einen wenig spektakulären Architektenentwurf.

An dem Architektenwettbewerb, der im Frühjahr I 852 ausgeschrieben wurde, beteiligten sich u. a. Joseph Paxton, der den Londoner Kristallpalast entworfen hatte und James Bogardus, ein zeitgenössischer Pionier der Glas-EisenKonstruktion. Bogardus hatte einen besonders innovativen und wagemutigen Vorschlag erarbeitet - eine dem Kolosseum ähnliche Konstruktion, die zergliedert werden konnte und mit einem Hängedach versehen war. Der Scientific American favorisierte diesen Vorschlag und bezeichnete ihn als »the best in every respect - in beauty, grandeur, originality, strength, simplicity, and economy «. ${ }^{78}$ Bogardus kam leider dennoch nicht zum Zuge. Sein Entwurf wurde vom zuständigen Auswahlgremium als »untried experiment « abgelehnt. Ausgewählt wurde schließlich der Vorschlag zweier relativ unbekannter New Yorker Architekten, George Carstensen, der den Tivoli Garten in Kopenhagen entworfen hatte, und Charles Gildemeister, die der Auswahlkommission zufolge die Kriterien »safety, simplicity and economy of construction; convenience of the visitors; a minimum of I 50000 square feet of exhibition space, and finally sarchitectural effect « ${ }^{79}$ am besten erfüllten. Carstensens Ent-

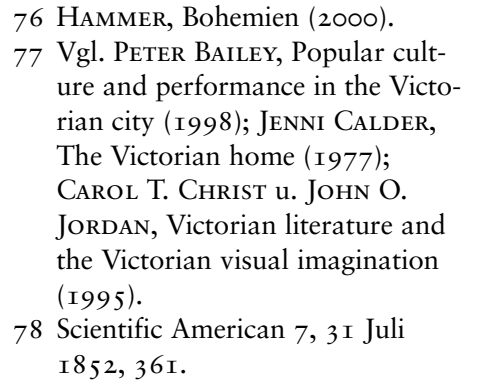

76 HAMmER, Bohemien (2000). ure and performance in the Victorian city (I998); JENNI CALDER, The Victorian home (I977) JORDAN, Victorian literature and the Victorian visual imagination Scientific American 7, 3 I Jul

Association, No. 53, in: American Phrenological Journal, I7.I.I 853. 25. August I852, Crystal Palace Mss, Holdings of the New York Historical Society. Das Gebäude umfasste dann tatsächlich eine Ausstellungsfläche von insgesamt I73,000 square feet, oder $\mathrm{I} 6074 \mathrm{~m}^{2}$. Davon entfielen IO 3 I $5 \mathrm{~m}^{2}$ auf das Erdgeschoss und $5759 \mathrm{~m}^{2}$ auf die Galerien. $\mathrm{Vgl}$. Bericht des Office of the 
wurf orientierte sich sehr stark am Londoner Vorbild. Er war im Grunde genommen eine Replik des Londoner Kristallpalastes in der Form eines griechischen Kreuzes, ergänzt um die obligatorische amerikanische Kuppel. ${ }^{8 \circ}$ Nach Auffassung des Architekturhistorikers Siegfried Giedion war das Gebäude »mediocre « und von keinerlei architekturhistorischer Bedeutung. ${ }^{8 \mathrm{I}}$

Bedeutsam war das Design jedoch unter transfergeschichtlichen Gesichtspunkten. In der Architektur des Londoner Kristallpalastes wurden die Gegensätze von Stadt und Land, von Fortschritt und Modernität einerseits und agrarischer Tradition andererseits, versöhnt, wenn nicht gar überwunden. Insofern schlossen die Organisatoren der New Yorker Ausstellung nicht nur an die symbolträchtige Architektur

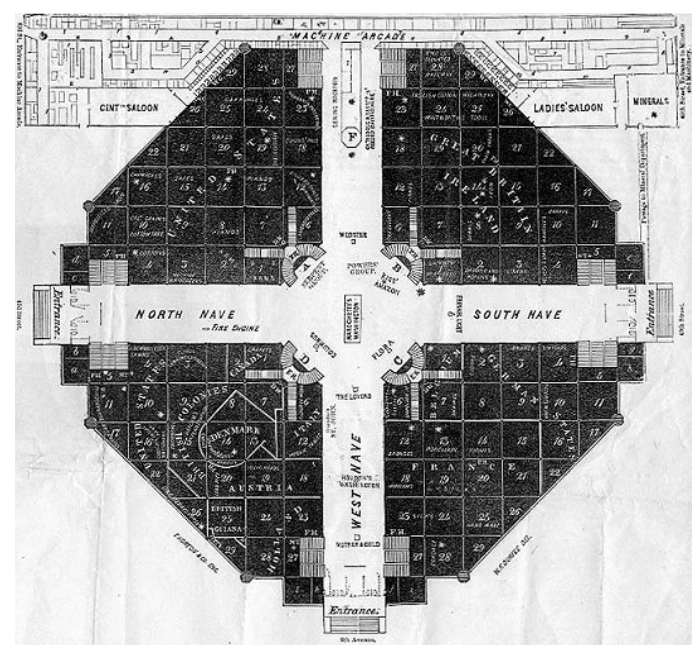

Abb. 3: Grundriss des New Yorker »Kristallpalastes «. Q: Guide to the Revised Official Catalogue of the Exhibition of the Industries of All Nations. The map was prepared by Henri C. Stuart and published by G. P. Putnam and Co. for the use of visitors to the New York Crystal Palace. des Londoner Kristallpalastes an, sondern auch an eine bestimmte Repräsentationsform dieser Architektur, die die bukolische Idylle mit der baulichen Utopie in Einklang brachte und damit die soziokulturellen Spannungen des StadtLand-Gegensatzes symbolisch aufhob. Dies wird insbesondere an den zeitgenössischen Lithographien deutlich, die den New Yorker Kristallpalast in einer Weise darstellten, die eine verblüffende Ähnlichkeit zu den Darstellungen des Londoner Kristallpalastes aufweist und kaum Referenzen zum New Yorker Standort und den dort vorhandenen Gebäuden enthält (vgl. Abb. 4 und 5).

Die Glas-Eisen-Konstruktion stellte bereits für die britischen Konstrukteure eine große technische Herausforderung dar. Der Londoner Kristallpalast bestand aus Fertigteilen, die vor Ort miteinander verbunden wurden. Obwohl Großbritannien Mitte des I9. Jahrhunderts zu den Eisen exportierenden Ländern zählte, gab es Engpässe bei der Lieferung der erforderlichen Baumaterialien. Angesichts veralteter Produktionsbedingungen und einer insgesamt nicht sehr bedeutenden Eisenproduktion muss der Bau des Kristallpalastes in New York nicht nur als Meilenstein in der amerikanischen Geschichte modernen Bauens und der Nutzung moderner Materialien herausgestellt werden, sondern auch als eine enorme materielle Leistung. ${ }^{82}$ Die New York Times apostrophierte den Bau der Ausstellungshalle deshalb auch als Ausdruck des Fortschrittsgeistes, der die amerikanische Gesellschaft erfasst habe und beherrsche: »The building now in course of construction in ReservoirSquare has been conceived on a scale, and is being forwarded with a dispatch and steadiness, commensurate with the great importance of the object and the spirit of progress, of which our country's name forms a synonyme. ${ }^{83}$
80 Für eine detaillierte zeitgenössische Beschreibung des Bauwerks vgl. The National Magazine 2, No. I, January I 853,80 .

8i Siegrried Giedion, Space, Time, and Architecture (I954).

82 Es wurden insgesamt I 250 Tonnen Eisen und $3624 \mathrm{~m}^{2}$ Glas verbaut. Vgl. Merry's Museum and Parley's Magazine I, January

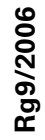
I 853,63 .
83 New York Times, 5. Oktober I 8 2; vgl. auch The National Magazine, Vol. 2, No. I, January I 853 , das den amerikanischen Kristallpalast mit St. Paul's Cathedral in London und dem Petersdom in Rom vergleicht und dabei dem Leser erläutert, dass St. Paul's deutlich kleiner ist als der New Yorker Kristallpalast und der Petersdom immerhin nur ein
Fünftel größer sei als das Weltausstellungsgebäude. 
Noch I 860 dominierte die baumwollverarbeitende Industrie mit insgesamt II4955 Beschäftigten den amerikanischen Industriesektor. In der Eisen- und Stahlproduktion waren demgegenüber lediglich 48975 Arbeiter beschäftigt. $^{84}$ Bis zur Mitte des 19. Jahrhunderts war Holz der primäre Baustoff. Eisen wurde bis zum Bürgerkrieg überwiegend aus Großbritannien importiert. Vor allem Eisenbahnschienen stammten aus britischer Produktion. ${ }^{85}$ Erst in den späten I850er Jahren hatte die einheimische Eisenproduktion ein Volumen erreicht, das den Ersatz der Importe durch heimische Produkte erlaubte. Die moderne Hochofentechnik auf der Basis des Bessemer-Verfahrens, das I8 55 patentiert worden war und die Eisenproduktion revolutionierte, wurde allerdings erst nach dem Bürgerkrieg eingeführt. ${ }^{86}$ Für den Bau des New Yorker Kristallpalastes wurden 190 achteckige Eisenpfeiler in einer Länge von 6,4 Metern im Erdgeschoss und I40 Eisenpfeiler in etwa der gleichen Größe im ersten Stock benötigt. Die

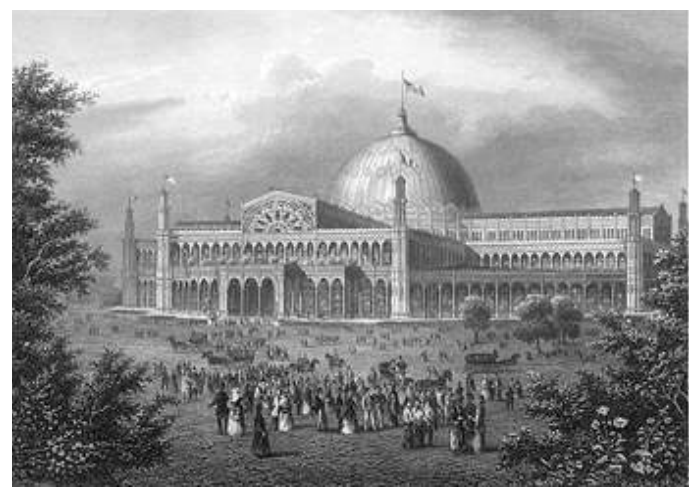

Abb. 4: Der New Yorker Kristallpalast.

Q: Carl Emil Doepler, Lithographie, in: New York Crystal Palace for the exhibition of the industry of all nations, designed by Carstensen \& Gildemeister N.Y. I 852 ; Library of Congress, Washington.

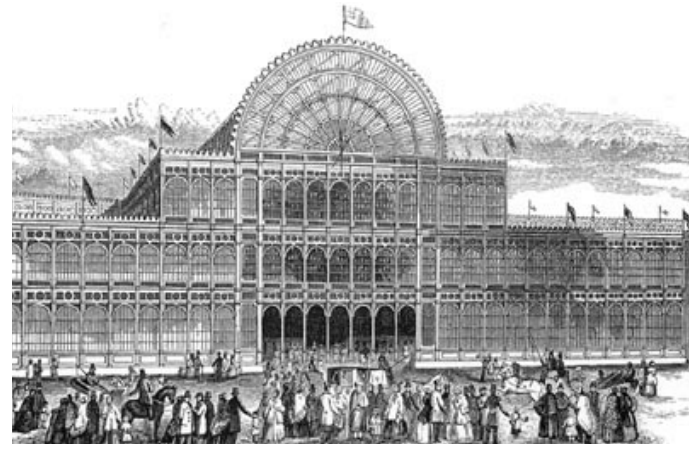

Abb. 5: Der Crystal Palace in London.

Q: R. Ackermann, Lithographie, in: The Park and Crystal Palace, London I85 I; Library of Congress prints.

Befestigung der 38 Meter hohen Kuppel, die einen Durchmesser von 30 Metern hatte, erforderte Eisenpfeiler in einer Länge von 2r Metern. ${ }^{87}$ Dieses Material wurde aus allen Teilen des Landes zusammengetragen. Obwohl die USA ein politisch und wirtschaftlich fragmentiertes Gebilde waren, demonstrierten sie mit dem Bau des Kristallpalastes, dass sie durchaus zu Leistungen imstande waren, die nur in einer nationalen Anstrengung, durch die Überwindung regionaler Gegensätze und regionalen Konkurrenzdenkens, bewerkstelligt werden konnten. In dieser Hinsicht entfaltete die Weltausstellung in New York nationale Selbstverständigungsprozesse, deren Bedeutung für die wirtschaftliche und industrielle Entwicklung der USA kaum überschätzt werden können.

Dazu gehörte auch ein sich im Zusammenhang mit der Ausstellungsarchitektur entwickelnder politischer Diskurs, der auf zentrale Elemente amerikanischer Selbstbeschreibungsmodelle des I9. Jahrhunderts zurückgreift. Dem demokratischen Ideal der jungen Republik folgend und in Abgrenzung von der aristokrati-

84 Robert Christian Puth, American economic history (I993) 217 .

85 Ibid., 216.

86 Ibid., 219.

87 Vgl. Godey' Lady's Book, Februar I 853 , I08. Umrechnung der Längen- und Raummaße erfolgt nach den US survey measures: $\mathrm{I}$ foot $=$ o,3048 Meter. 
schen Tradition des Londoner Kristallpalastes sollte der New Yorker Kristallpalast ein »Palace of the People ${ }^{88}{ }^{8}$ werden. Um dies sicherzustellen, hatte bereits das Board of Alderman in seiner Bewilligung des Baus festgelegt, dass der Eintrittspreis für Einzelbesucher zu keiner Zeit den Betrag von 50 Cents überschreiten dürfe. ${ }^{89}$ Mehr als sein Londoner Vorbild sollte der New Yorker Kristallpalast die Menschen aller Klassen und auch aller (amerikanischen) Regionen zusammenbringen. So erläuterte etwa ein Beobachter im DeBow's Review of the Southern and Western States im Dezember I 852:

»The enterprise proposes to bring together the men of all these regions into one great conclave; side by side the woodcutter of Maine and the miner of the Sacramento; the hardy whaleman of Nantucket, and the stout boatman of the Illinois; the caravan driver of Santa Fe, and the frontier man of Nebraska; the manufacturer of Lowell; the tobacco grower of the Roanoke, and the hemp and wheat sower of Missouri and the lakes; the merchant princes of New York; the polished and wealthy planters of the Ashepoo, the Alabama, the Lafourche, and the Attakapas; the brave and sturdy hunters of Texas and Arkansas. «9०

Der Kristallpalast entwickelte sich in der zeitgenössischen Berichterstattung zum Symbol einer demokratischen Kultur der »unbegrenzten Möglichkeiten «. Was jedoch diese demokratische Kultur im Einzelnen ausmachte und beinhaltete, darüber war man sich in der Mitte des I9. Jahrhunderts noch keineswegs einig. Humbug und Kuriositäten, Wettbuden und Hahnenkämpfe zählten jedenfalls ganz offensichtlich ebenso dazu wie Gemälde, Skulpturen und technische Attraktionen. ${ }^{9 \mathrm{I}}$ Der New Yorker Kristallpalast steht deshalb auch als Metapher für kulturelle Ambivalenzen und Unsicherheit.
Während die Repräsentation des demokratischen Ideals in Form von side shows vom New Yorker `Bürgertum`, das seine eigenen quasiaristokratischen Traditionen entwickelt hatte, abgelehnt wurde, kritisierten die intellektuellen 'Demokraten die Übernahme aristokratischen Verhaltens. So kommentierte der Scientific American das Eröffnungszeremoniell, mit dem die Mitglieder der Association sowie die geladenen Vertreter aus der Politik durch die Übertragung des Protokolls der Londoner Eröffnungsfeier ganz offensichtlich das Ziel verfolgten, sich über Performanz zu nobilitieren, ${ }^{92}$ mit der bitteren Aussage: "Our country is not yet republican in spirit. « Und der Kommentar ergänzte: » it is only so in name, and the opening of the Crystal Palace afforded full proof of the truth of what we say. It is a political aristocracy: petty squires, secondrate lawyers, caponlined Aldermen, hairy-faced men with epauletts [sic] on their shoulders, and such-like characters, were treated with scome up here, there are chief seats for you<. Distinguished inventors, artists, engineers, and mechanicians, the men who should have been most prominent were treated with sit down there - see there are some footstools for you $\cdot \ll{ }^{93}$

\section{Schlussbetrachtung}

Das Scheitern der Weltausstellung von I 853 ist ein Beispiel für Dysfunktionalitäten, die sich bei kulturellen Transfers aufgrund von asynchronen historischen Kontextbedingungen ergeben. Die Übertragung des europäischen Konzeptes , Weltausstellung erfolgte zu früh. Die junge Republik war kulturell, infrastrukturell und im Hinblick auf die Organisation des politischen Prozesses sowie des Prozesses der Nationenbildung noch nicht bereit, eine derartige Veranstaltung auszurichten. Während in Europa mit
88 New York Times, 20. Mai, 20. Juni i 853 ; Horace Greeley (Hg.), Art and Industry (18 53) 30.

89 New York Daily Times, 26. Dezember I85 I. 50 Cents im Jahr I 853 entsprechen einer heutigen Kaufkraft von ca. I 2 Dollar.

90 DeBow's Review of the Southern and Western States, Vol. I, No. 6 (Dec. I 852) 637.

๕ั.

9I Zum Zusammenhang von Education und Entertainment auf den
Weltausstellungen vgl. PAUL GreEnHALGH, Ephemeral vistas (i988) 5, i9-2i; Тімотну MiтCHELL, The World as Exhibition (I989) 2I7-236.

92 Zum Begriff der perfomativen Politik vgl. Rahmenantrag des SFB 584 sowie Jan ANDrEs u. Matthias Schwengelbeck, Das Zeremoniell als politischer Kommunikationsraum (2005) 27-8I.
93 Scientific American III, No. 45, 23 July I 853 . 
den großen Ausstellungen in London ( 1862 ) und Paris (I855 und I867) das Ausstellungswesen florierte und in zunehmendem Maße die nationalistischen Aspirationen und die wirtschaftliche Entwicklung der Gastgeber widerspiegelte, kämpften die Amerikaner im Bürgerkrieg und versuchten anschließend ihre Nation wieder aufzubauen. Erst jetzt, Ende der I86oer und zu Beginn der I 870 er Jahre und damit 20 bis 30 Jahre später als in Europa, waren die Anfänge der industriellen Revolution in den USA spürbar und begann man das wirtschaftliche, soziale und kulturelle Ausmaß dieses zentralen Prozesses des I9. Jahrhunderts zu erahnen. Im Innern wurde die Industrie zum beherrschenden Sektor, und im Weltzusammenhang rückten die USA von der Peripherie des kapitalistischen Systems allmählich näher zum Zentrum. Durch den Bevölkerungszuwachs und die verkehrsmäßige Erschließung des Kontinents kam nun erst die Tatsache voll zum Tragen, dass die USA über einen riesigen Binnenmarkt verfügten, der - anders als in Europa - nicht durch politische Grenzen und Zollschranken behindert wurde.

Das ursprünglich in Frankreich Ende des I8. Jahrhunderts entstandene Konzept der Industrieausstellung entwickelte sich deshalb erst im letzten Drittel des I9. Jahrhunderts, im Gilded Age und der Victorian Era, durchaus in Konkurrenz zu den großen europäischen Ausstellungsnationen - Großbritannien und Frankreich - zu einer amerikanischen Institution. ${ }^{94}$ In einem engen transatlantischen Dialog vollzog sich dabei ein konzeptioneller Wandel in der Ausstellungsidee, der sich nicht zuletzt semantisch niederschlug: Weltausstellungen hießen in Großbritannien World exhibitions, während das französische Äquivalent exposition universelle lautete. In den USA nannte man sie World's fairs - eine Bezeichnung, die die sich seit der Wende zum 20. Jahrhundert immer stärker abzeichnende Entwicklung des amerikanischen Ausstellungswesens hin zu einer Mischung aus education und entertainment im Sinne des modernen Edutainment-Konzeptes begrifflich vorwegnahm. Seinen Anfang nahm dieser Prozess in New York I 853, in den Seitenflügeln des Kristallpalastes. Bereits die zweite Weltausstellung auf amerikanischem Boden, die I876 in Philadelphia stattfand, muss in diesem Sinne als ramerikanische Ausstellung bezeichnet werden. Die Columbian Exposition schließlich, die I 893 zum Gedenken an die Entdeckung Amerikas durch Kolumbus in Chicago durchgeführt wurde, ${ }^{95}$ ließ im Vergleich zu den großen europäischen Ausstellungen nichts an nationaler Rhetorik und Symbolik vermissen. Sie bediente den Fortschrittsdiskurs ebenso wie das Bedürfnis nach Massenunterhaltung. Der neue Reichtum der Unternehmer und Wirtschaftsmagnaten wurde zur Schau gestellt, und zwar »conspicuously ", wie Thorsten Veblen es nannte. ${ }^{96}$ Der Prestigekonsum des viktorianischen Zeitalters - so das Argument von Rydell - bildete die Basis für »the Victorian era's most extravagant exhibitionary form - the world's fair «. ${ }^{97}$

Einleitend ist bereits auf die Notwendigkeit hingewiesen worden, die Geschichte der europäisch-amerikanischen Beziehungen als Geschichte des kulturellen Transfers mit ganz eigenen Aneignungs- und Abwehrprozessen zu schreiben. Der hier präsentierte Versuch einer empirischen Umsetzung dieser Perspektive hat indes ein zweites Desiderat deutlich gemacht, das in der bisherigen Methodendebatte zu wenig Beachtung gefunden hat. Das Aufspüren und die Erfassung der sich über kulturelle Transfers herausbildenden 'feinen Unterschiede erfordert eine gründliche Kenntnis nicht nur der kulturellen und politischen Traditionen der Ursprungs-

94 Rydell, Findling u. Pelle, Fair America (2000) I.

95 Robert W. Rydell, All the world's a fair (I984); RYDELL, Findling u. Pelle, Fair America (2000).

96 Thorstein Veblen, The Theory of the Leisure Class (I899).

97 Rydell, Findling u. Pelle, Fair America (2000) I9. 
gesellschaften, sondern auch der Geschichte der Empfängergesellschaften. Aus diesem Grund ist Regionalkompetenz eine unhintergehbare Voraussetzung für seriöse Transfergeschichte. Dabei fällt auf, dass in der gegenwärtigen Debatte um die Wiederbelebung der Regionalstudien »Amerika « (d.h. die Vereinigten Staaten und Kanada) systematisch ausgeblendet wird. ${ }^{98}$ Die Debatte bezieht sich allein auf »exotische « Regionen: den Nahen und Mittleren Osten, Afrika, Asien und auch Lateinamerika. Die Beschäftigung mit den Inhalten und Kompetenzen, die ein Area-Spezialist besitzen muss, blendet dabei aus, dass Fremdheit nicht allein über fremde Sprachen konstituiert wird.

Die Ausblendung "neo-europäischer " Gesellschaften aus der gegenwärtigen Debatte um die Revitalisierung der Regionalstudien zeugt von einer eurozentrischen Perspektive, durch die insbesondere die Kulturgeschichte "Amerikas « unter die europäische oder besser abendländische Tradition subsumiert wird. Diese Subsumption fördert die Tendenz, politisch-kul- turelle Unterschiede und Asynchronizitäten auszublenden. Dies verstellt den Blick für Dysfunktionalitäten, die beim Versuch der Übernahme europäischer Institutionen im amerikanischen Kontext erzeugt werden. Solche Dysfunktionalitäten sind aber häufig Ausgangspunkt von historischen Eigenentwicklungen, die es aufzuspüren gilt. Das Scheitern der > Weltausstellung < in New York 1853 und die Amerikanisierung der Institution >Weltausstellung im späten 19. und frühen 20. Jahrhundert sind prägnante Beispiele dafür. Bereits I 853 wurden vor dem Hintergrund sich deutlich von Europa unterscheidender historischer Kontextbedingungen neue Muster generiert, die dann ihrerseits, im Sinne der histoire croisée, ${ }^{99}$ auf die Ausgangskulturen zurückwirkten. Scheitern - dies hat die vorliegende Analyse gezeigt - ist insofern aus der Perspektive der Transferforschung und der histoire croisée eine zentrale historische Kategorie.

\section{Ursula Lehmkuhl}

\footnotetext{
98 Vgl. hierzu die Positionspapiere des Wissenschaftsrats, z. B. das "Freiburger Memorandum zur Zukunft der Regionalstudien in Deutschland unter besonderer Berücksichtigung Asiens, Afrikas, Lateinamerikas und des Nahen Ostens « vom November 2005 (http://www.daad.de/de/download/ hochschulen/veranstaltungen/ kulturwissenschaften 2005

๕ั memorandum.pdf); sowie Kon-
}

zept und Vorträge der vom Wissenschaftskolleg im Rahmen des Forschungsschwerpunktes »Wege des Wissens « organisierten Konferenz »Zukunft der Regionalstudien « vom Juli 2005 (http:// www.wiko-berlin.de/kolleg/ projekte/wegedw/wdwarbeit ber?hpl=I). Das gleiche gilt auch für die amerikanische Diskussion. Vgl. hierzu das Programm der Ford Foundation »Crossing Bor- ders. Revitalizing Area Studies«, I999.

99 Michael Werner u. Bénédicte ZimmermanN, Vergleich, Transfer, Verflechtung (2002). 
Literatur:

Lynn Аввott, Doug Seroff (2007), Ragged but right. Black traveling shows, "coon songs", and the dark pathway to blues and jazz, I. Aufl., Jackson.

Jan Andres, Matthias SchwengelBECK (2005), Das Zeremoniell als politischer Kommunikationsraum. Inthronisationsfeiern in Preußen im >langen I9. Jahrhundert, in: Neue Politikgeschichte. Perspektiven einer historischen Politikforschung, hg. von UTE Frevert, HeinZ-Gerhard Haupt, Frankfurt a. M., New York, 27-8I.

Catherine M. ANDronik (I994), Prince of Humbug. A life of P. T. Barnum, I. Aufl., New York, Toronto.

Peter Bailey (1998), Popular culture and performance in the Victorian city, Cambridge UK, New York.

P. T. BARnum (I855), The life of P. T. Barnum, Author's ed., London.

P. T. Barnum, George S. Bryan (I927), Struggles and triumphs. Or, The life of P.T. Barnum, New York, London.

Annemarie Bean, James Vernon Hatch, Brooks McNamara (I996), Inside the minstrel mask. Readings in nineteenth-century blackface minstrelsy, Hanover NH.

Sven Beckert (200I), The Monied Metropolis. New York City and the Consolidation of the American Bourgeoisie, I $850-$ I 896, Cambridge UK, New York NY.

Burton Benedict (I99I), International exhibitions and national identity, in: Anthropology Today 7 , 5-9.

David Harry Bennett (1995), The party of fear. From nativist movements to the New Right in American history, 2. überarb. und akt. Vintage Books Aufl., New York.

Tony Bennetr (I988), The Exhibitionary Complex, in: New Formations 4, 73-IO2.

WOLFGANG BERgSDORF, Universität Erfurt, Fachhochschule Erfurt, Thüringische Landeszeitung (Weimar Thuringia Germany), Sparkassen- und Giroverband HessenThüringen (2003), Amerika, fremder Freund. I I Vorlesungen, I. Aufl., Weimar.
M. Christine Boyer (I985), Manhattan Manners. Architecture and Style i $850-$-1900, New York.

Asa Briggs (I968), Victorian Cities, London.

J. BRYAN (I956), The world's greatest showman. The life of P. T. Barnum, New York NY.

Michael Burgan (2006), A changing nation. Making a new nation, Chicago.

Jenni Calder (I977), The Victorian home, London.

Donald James Cannon (1976), The Fire Department of the City of New York, I835-I898. A Study in Institutional Adaptibility, Fordham University.

Georg Carstensen, Karl GildeMEISTER (I 854 ), New York Crystal Palace. Illustrated description of the building, New York.

Tertius Chandler (I987), Four thousand years of urban growth. An historical census, Lewiston NY.

Carol T. Christ, John O. Jordan (I995), Victorian literature and the Victorian visual imagination, Berkeley.

Sebastian Conrad, Jürgen OsterHAMMEL (Hg.) (2004), Das Kaiserreich transnational. Deutschland in der Welt I87I-I9I4, Göttingen.

Sebastian Conrad, Shalini RandeRIA (2002), Geteilte Geschichten Europa in einer postkolonialen Welt, in: Jenseits des Eurozentrismus. Postkoloniale Perspektiven in den Geschichts- und Kulturwissenschaften, hg. von SEBASTIan ConRad, Shalini Randeria, Frankfurt a. M., New York, 9-49.

Donald R. Davis, Federal Reserve Bank of New York (I997), The home market, trade, and industrial structure, Federal Reserve Bank of New York [cited, available from http://www.ny.frb.org/ research/staffreports/sr3 3. html].

Mona Domosh (I996), Invented cities. The creation of landscape in nineteenth-century New York, Boston, New Haven.

Felix Driver, David Gilbert (I999), Imperial cities. Landscape, display and identity, St. Martin's Press, Manchester, New York.
Michel Espagne (2000), Kulturtransfer und Fachgeschichte der Geisteswissenschaft, in: Comparativ IO, no. I, 42-6I.

Michel Espagne (1999), Les transferts culturels franco-allemands, Paris.

Andreas Etges (I999), Wirtschaftsnationalismus. USA und Deutschland im Vergleich (I8I5-I9I4), Frankfurt a. M., New York.

Alice Mulcahey Fleming (I993), P. T. Barnum. The world's greatest showman, New York.

DAN B. Fleming JR. (2004), A West Virginia Boy at the New York World's Fair, Goldenseal 30, no. 2, IO.

Celina Fox (I992), London - World City. I 800-I 840, New Haven.

Etienne François, Hagen Schulze (200I), Deutsche Erinnerungsorte, 3 Bde., München.

Ute Frevert, Heinz-Gerhard Haupt (2005), Neue Politikgeschichte. Perspektiven einer historischen Politikforschung, in: Historische Politikforschung, Bd. I, Frankfurt a. M., New York.

Alexander C. T. Geppert (2004), London vs. Paris. Imperial Exhibitions, Transitory Spaces, and Metropolitan Networks. I8801930, European University Institute.

Siegfried Giedion (I954), Space, Time, and Architecture. The Growth of a New Tradition, in: The Charles Eliot Norton lectures for 1938-1939, 3. Aufl., Cambridge.

Stephen F. Ginsberg (I97I), The Police and Fire Protection in New York City: I800-I 850 , in: The Quarterly Journal of the New York State Historical Association 52, no. I, I33-I 50 .

Horace Greeley (Hg.) (I 853 ), Art and Industry as Represented in the Exhibition at the Crystal Palace, New York, I853-54, New York.

Paul Greenhalgh (I988), Ephemeral vistas. The expositions universelles, great exhibitions, and world's fairs, I 85 I-I939, Manchester UK, New York.

Markus GüNTHer (2005), Auf dem Weg in die Neue Welt. Die Atlantiküberquerung im Zeitalter der Massenauswanderung I 8 I 8I9I4, Augsburg. 
UtZ Haltern (I973a), Die »Welt als Schaustellung «. Zur Funktion und Bedeutung der internationalen Industrieausstellung im I9. und 20. Jahrhundert, in: Vierteljahresschrift für Sozial- und Wirtschaftsgeschichte 60, I-40.

Utz Haltern (1973b), Die Londoner Weltausstellung von I 85 I. Ein Beitrag zur Geschichte der bürgerlich-industriellen Gesellschaft im I9. Jahrhundert (Neue Münstersche Beiträge zur Geschichtsforschung im I9. Jahrhundert, Bd. I3), Münster.

Klaus Hammer (2000), Bohemien, Astralhumorist, Katerpoetiker, Architekturphantast und Geschichten-Erfinder Paul Scheerbart - ein Utopist der Jahrhundertwende, in: Orbis Linguarum I 5 , I-6.

Evelynn Maxine Hammonds (I999), Childhood's Deadly Scourge. The Campaign to Control Diphtheria in New York City, I880-1930, Baltimore.

George D. Harmon (I929), Aspects of slavery and expansion, I $848-$ 60 , Bethlehem.

Klaus Harpprecht (1982), Der fremde Freund. Amerika, eine innere Geschichte, Stuttgart.

John Higham (2002), Strangers in the land. Patterns of American nativism, I860-I925, New Brunswick NJ.

Charles Hirschfeld (i957), America on Exhibition. The New York Crystal Palace, in: American Quarterly 9, no. 2, IOI-II6.

Hermione Hobhouse (2004), The Crystal Palace and the Great Exhibition. Art, science, and productive industry. A history of the Royal Commission for the Exhibition of 185 I, London, New York.

Hermione Hobhouse (I97I) Thomas Cubitt. Master builder, London.

Dirk Hoerder (2002), Cultures in contact. World migrations in the second millennium, Comparative and international working-class history, Durham.

Peter H. Hoffenberg (200I), An empire on display. English, Indian, and Australian exhibitions from the Crystal Palace to the Great War, Berkeley.

Eric Homberger (I994), The historical atlas of New York City.
A visual celebration of nearly 400 years of New York City's history, New York.

Linda Hyman (I974), Crystal Palace/ 42 Street/I 853-54, New York.

Thomas JefFerson (I984), Writings, New York.

Charles A. Jones (1987), International business in the nineteenth century. The rise and fall of a cosmopolitan bourgeoisie, New York.

WOLFRAM KAISER (2000), Die Welt im Dorf. Weltausstellungen von London I 85 I bis Hannover 2000, in: Aus Politik und Zeitgeschichte B22-23, no. 26. Mai 2000, 3-IO.

Dale T. Knobel (i996), America for the Americans. The nativist movement in the United States. Social movements past and present, New York, London.

Winfried Kretschmer ( I999), Geschichte der Weltausstellungen, Frankfurt am Main, New York.

Dieter LANGEWIESCHE (2000), Nation, Nationalismus, Nationalstaat in Deutschland und Europa, München.

Judith Walzer Leavitt (i996), Typhoid Mary. Captive to the Public's Health, Boston.

Ursula LehmKuhl (2005), Creating Anglo-American Friendship. The Great Exhibition of I 85 I and the Social Construction of the Special Relationship, in: From Enmity to Friendship. Anglo-American Relations in the I9th and 2oth Century, hg. von URSULA LeHMKUHL, Gustav Schmidt, Augsburg, 27$5 \mathrm{I}$.

URSULA LeHMKUHL (2004), Una mietitrice come catalizzatore. La Great Exhibition del I 85 I e la costruzione sociale della relazione speciale anglo-americana, in: Esposizioni in Europa tra otto e novecento. Spazi, organizzazione, rappresentazioni, hg. von C. T. Geppert, Massimo Baioni, Mailand, I4I-64.

Lars Magnusson (I996), Free trade I793-I 886. Early sources in economics, London, New York.

Roland Marchand (I99I), Corporate Imagery and Popular Education. World's Fairs and Expositions in the United States, I893-I940, in: European Contributions to American Studies, no. $2 \mathrm{I}$, I 8 .
SARAh MeER (2005), Uncle Tom mania. Slavery, minstrelsy, and transatlantic culture in the $1850 \mathrm{os}$, Athens.

ILJA MiecK (I998), Deutschland und die Pariser Weltausstellung, in: Deutsch-französischer Kulturtransfer im europäischen Kontext, hg. von Etienne François, M. C. Hoock-Demarle, R. Meyer-Kalkus, Michael WERNER, Leipzig.

Timothy Mitchell (I989), The World as Exhibition, in: Comparative Studies in Society and History 3I, no. 3, 217-236.

Jerome MushKat (i98I), The reconstruction of the New York democracy, I86I-I 874, Rutherford NJ, London.

Jerome MushKat (I97I), Tammany. The evolution of a political machine. I789-I865, I. Aufl., Syracuse NY.

Mark E. Neely (2005), The boundaries of American political culture in the Civil War era, The Steven and Janice Brose lectures in the Civil War era, Chapel Hill.

Pierre Nora (1984), Les Lieux de mémoire. Bibliothèque illustrée des histoires, Paris.

Official catalogue of the New York exhibition of the industry of all nations (I853), I. verb. Aufl., New York.

Jürgen Osterhammel (200I), Geschichtswissenschaft jenseits des Nationalstaats. Studien zu Beziehungsgeschichte und Zivilisationsvergleich, Göttingen.

Jürgen Osterhammel (2003), Transferanalyse und Vergleich im Fernverhältnis, in: Vergleich und Transfer. Komparatistik in den Sozial-, Geschichts- und Kulturwissenschaften, hg. von HARTMUT KAELbLe, JÜrgen SCHRIEWER, Frankfurt a. M., New York, 439468.

Johannes Paulmann (I998), Interkultureller Transfer zwischen Deutschland und Großbritannien. Einführung in ein Forschungskonzept, in: Aneignung und $\mathrm{Ab}-$ wehr. Interkultureller Transfer zwischen Deutschland und Großbritannien im I9. Jahrhundert, hg. von Rudolf Muhs, Johannes Paulmann, Willibald SteinMETZ, Bodenheim, 2I-43. 
Johannes Paulmann (I998), Internationaler Vergleich und interkultureller Transfer. Zwei Forschungsansätze zur europäischen Geschichte des I 8. bis 20. Jahrhunderts, in: Historische Zeitschrift 267, 649-685.

Selim H. Peabody, YA Pamphlet Collection (Library of Congress) (I902), World's fairs. Universal expositions the milestones along the highway of human progress, St. Paul Minn.

ROBert C. Post (I976), ,Liberalizers versus 'Scientific Men in the Antebellum Patent Office, in: Technology and Culture I6, 24-54.

Robert C. Post (1983), Reflections of American Science and Technology at the New York Crystal Palace Exhibition of 1853 , in: Journal of American Studies I7, no. 3, 337356.

Robert Christian Puth (I993), American economic history, 3. Aufl., Fort Worth.

Thomas Laurence RiIs, Brooklyn College. Institute for Studies in American Music (1992), More than just minstrel shows. The rise of black musical theatre at the turn of the century, in: I.S.A.M. monographs, no. 33, Brooklyn NY.

C.T. Rodgers (I 852 ), American Superiority at the World's Fair, Philadelphia.

NaOmi Rogers (I992), Dirt and Disease. Polio Before FDR, New Brunswick NJ.

Robert Rydell (1983), Visions of Empire. International Expositions in Portlant and Seattle, I905I909, in: Pacific Historical Review 52, no. I, 37.

Robert W. Rydell (1984), All the world's a fair. Visions of empire at American international expositions, I876-I9I6, Chicago.
Robert W. Rydell (I988), The Open (Laboratory) Door. Scientists and Mass Culture, in: European Contributions to American Studies I3, 6I.

Robert W. Rydell (1993), World of fairs. The century-of-progress expositions, Chicago Ill.

RoBERT W. RYDELL, JoHN E. Findling, Kimberly D. Pelle (2000), Fair America. World's Fairs in the United States, Washington DC.

SASKIA SASSEN (1994), Cities in a world economy. Sociology for a new century, Thousand Oaks Calif.

Saskia SASSEn (200I), The global city, New York, London, Tokyo, 2. Aufl., Princeton NJ.

SASKIA SASSEN (2002), Global networks. Linked cities, New York.

Ronald E. Seavoy (2006), An economic history of the United States. From I607 to the present, New York.

Benjamin Silliman JR., C. R. GoodRICH (Hg.) (I 854), The World of Science Art and Industry Illustrated from Examples in the New-York Exhibition I $853-$ I854, New York.

WARREN SLOAT (2002), A battle for the soul of New York: Tammany Hall, police corruption, vice, and Reverend Charles Parkhurst's crusade against them, I892-I895, I. Cooper Square Press Aufl., New York.

Anthony D. Smith (I987), The ethnic origins of nations, Oxford UK, New York.

Anthony D. SMith (200I), Nationalism. Theory, ideology, history, Malden Mass.

Anthony D. Smith (1983), Theories of nationalism / Anthony D. Smith, 2. Aufl., New York NY,

IVAN STEEN (I 999), New York I 853 , in: Historical Dictionary of World's
Fairs and Expositions, I $85 \mathrm{I}-$ I988, hg. von John E. Findling, Kimberly D. Pelle, i2. Westport Conn.

Ivan D. Steen (1963), America's First World's Fair, in: New-York Historical Society Quarterly 47, no. 3 , 256-280.

ROBERT C. TOLL (I974), Blacking up. The minstrel show in nineteenth century America, New York.

David Van Veersbilck, Peter DiSante, Brian Mark, Roger Smith, Vincent Tufo, Percy Danforth, Matthew HeuMANN, ROBERT B. Winans, Recorded Anthology of American Music Inc. (I985), The Early minstrel show, New York NY, sound recording.

Thorstein Veblen (i 899), The Theory of the Leisure Class. An Economic Study in the Evolution of Institutions, New York, London.

Joe Vitale (2006), There's a customer born every minute. P. T. Barnum's amazing Io "rings of power " for creating fame, fortune, and a business empire today - guaranteed!, Hoboken NJ.

Michael Werner, Bénédicte ZimMERMANN (2002), Vergleich, Transfer, Verflechtung. Der Ansatz der Histoire croisée und die Herausforderung des Transnationalen, in: Geschichte und Gesellschaft 28, no. 4, 607-636.

Clint WiLlis (2003), Crimes of New York. Stories of crooks, killers and corruption from the world's toughest city, New York.

Martin Wörner (2000), Die Welt an einem Ort. Illustrierte Geschichte der Weltausstellungen, Berlin.

MARTIN WöRNER (I999), Vergnügung und Belehrung. Volkskultur auf den Weltausstellungen I 85 II900, München. 